COMMUNICATIONS IN

ANALYSIS AND GEOMETRY

Volume 2, Number 1, 1-33, 1994

\title{
UNIQUENESS OF SOME CYLINDRICAL TANGENT CONES
}

\author{
LEON SIMON*
}

It is known ([SL1]) that if a minimal submanifold $M$ has a tangent cone $\mathbf{C}$ at a singular point $p$, then $\mathbf{C}$ is the unique tangent cone of $M$ at $p$, and $M$ approaches $\mathbf{C}$ asymptotically in the appropriate smooth sense, provided that $\operatorname{sing} \mathbf{C}=\{0\}$ and $\mathbf{C}$ has multiplicity 1 .

It has remained an open question whether or not this carries over to the case when sing $\mathbf{C}$ consists of more than one point. Here we want to settle the question (in the affirmative) in certain of the cases when $\mathbf{C}$ is an $n$-dimensional cylinder in $\mathbf{R}^{n+1}$ with (n-1)-dimensional cross section $\mathbf{C}_{0} \subset \mathbf{R}^{n}$ satisfying the same conditions as those mentioned above for $\mathbf{C}$ (i.e., we treat certain cases when $\mathbf{C}$ has the form $\mathbf{C}=\mathbf{C}_{0} \times \mathbf{R}$, with $\operatorname{sing} \mathbf{C}_{0}=\{0\}$ and multiplicity of $\mathbf{C}_{0}=1$ at each point $\left.x \in \mathbf{C}_{0} \backslash\{0\}\right)$. In a suitable class of minimal hypersurfaces (to be described in detail below, and which includes the codimension one minimizing currents), we give conditions on the cross-section $\mathbf{C}_{0}$ of $\mathbf{C}$ which are sufficient to guarantee that $\mathbf{C}$ is the unique tangent cone whenever it arises as a mutiplicity one tangent cone at all.

The restrictions on $\mathbf{C}_{0}$ are that $\mathbf{C}_{0}$ be a strictly minimizing (in the sense defined in [HS]), strictly stable cone and that the Jacobi-field operator satisfy certain restrictions on its first few eigenvalues, as described in conditions $\ddagger(a)$, (b), (c) below. A brief discussion about which of the known area minimizing hypercones satisfy the conditions $\ddagger$ which are needed is given in Remark (2) following $\ddagger$ below; certainly all the cones over products of spheres satisfy the requirements in case $n>8$ - see the discussion following Theorem 1 of $\S 1$. It is interesting to note that the cones over $S^{3} \times S^{3}$ and $S^{2} \times S^{4}$ (corresponding

Research partly supported by NSF grants DMS-8703537 and DMS-9012718 at Stanford University. 
to the case $n=8$ ) do not satisfy the condition $\ddagger$ (c) needed in Theorem 1 , so the question of whether these are always unique tangent cones when they arise at all as multiplicity one tangent cones remains open.

The main technical ingredient in the present work is the result of Theorem 2.1, which gives a new $L^{2}$ estimate for stationary oriented hypersurfaces which are close to a cylindrical cone with strictly stable strictly minimizing cross-section.

We should point out here that while the recent work [SL5] establishes a.e. uniqueness of tangent cones for various classes classes of minimal surfaces, the results in [SL5] are of a different character to those obtained here; most notably the methods of [SL5] do not establish (as we do here) that certain tangent cones are always unique whenever they appear as tangent cones (of a minimal surface in a multiplicity one class) at all. Rather, the main unique asymptotic results of [SL5] are conditional on the a priori assumption that there are no "gaps" in the singular set near the singular point $p$ under consideration. (This is sufficient to establish rectifiability of the singular sets of various classes of minimal surfaces, which was the main point of [SL5].)

\section{Notation and Statement of Main Theorem}

$$
B_{\rho}=\left\{x \in \mathbf{R}^{n}:|x|<\rho\right\}, \quad B_{\rho}(y)=\left\{x \in \mathbf{R}^{n}:|x-y|<r\right\} .
$$

$\eta_{Y, \rho}$ will denote the transformation of $\mathbf{R}^{n+1}$ which translates $Y$ to 0 and homotheties by $\rho^{-1}$; thus $\eta_{Y, \rho}(X)=\rho^{-1}(X-Y)$.

$\mathbf{C}=\mathbf{C}_{0} \times \mathbf{R}$ will denote an $n$-dimensional cylinder in $\mathbf{R}^{n+1}$, with crosssection $\mathbf{C}_{0}$ an $(n-1)$-dimensional cone in $\mathbf{R}^{n}$ such that $\mathbf{C}_{0} \cap S^{n-2}$ is an embedded compact $(n-2)$-dimensional minimal submanifold $\Sigma$ of $S^{n-1}$.

We let $\mathcal{M}_{\mathbf{C}}, \mathcal{M}_{\mathbf{C}_{0}}$ and note the minimal surface operators (i.e. the EulerLagrange operators for the area functionals) on $\mathbf{C}$ and $\mathbf{C}_{0}$ respectively, and let $L_{\mathbf{C}}, L_{\mathbf{C}_{0}}$ be the linearizations of $\mathcal{M}_{\mathbf{C}}, \mathcal{M}_{\mathbf{C}_{0}}$ respectively at 0 . Thus

$$
\begin{aligned}
L_{\mathbf{C}} v & =\frac{\partial^{2} v}{\partial y^{2}}+L_{\mathbf{C}_{0}} \\
& =\frac{\partial^{2} v}{\partial y^{2}}+\triangle_{\mathbf{C}_{0}} v+\frac{q(\omega)}{r^{2}} v
\end{aligned}
$$




$$
=\frac{\partial^{2} v}{\partial y^{2}}+\frac{1}{r^{n-2}} \frac{\partial}{\partial r}\left(r^{n-2} \frac{\partial v}{\partial r}\right)+\frac{1}{r^{2}}\left(\Delta_{\Sigma} v+q(\omega) v\right),
$$

where $r^{-2} q(\omega)$ is the length of the second fundamental form of $\mathbf{C}_{0}$, as a submanifold of $\mathbf{R}^{n+1}$, at the point $r \omega \in \mathbf{C}_{0}$. The operator $\triangle_{\Sigma}+q(\omega)$ appearing here will henceforth be denoted $L_{\Sigma}$. Since $L_{\Sigma}$ is an elliptic operator on the smooth compact manifold $\Sigma$, the eigenvalues of $-L_{\Sigma}$ form a sequence

$$
\lambda_{1}<\lambda_{2} \leq \lambda_{3} \leq \ldots \leq \lambda_{k} \leq \ldots, \lambda_{k} \uparrow \infty
$$

and there is a corresponding orthonormal sequence of eigenfunctions

$$
\varphi_{1}, \varphi_{2}, \varphi_{3}, \ldots, \varphi_{k}, \ldots
$$

(orthonormal with respect to the $L^{2}(\Sigma)$-norm).

There is an important method of generating solutions of the equation $L_{\mathbf{C}_{0}} v=$ 0 , analogous to the method in Riemannian geometry of generating Jacobi fields by taking the initial velocity vector of a 1-parameter family of geodesics, as follows:

Suppose $\left\{M_{t}\right\}_{\{t \in(0,1)\}}$ is a 1-parameter family of minimal hypersurfaces in $\mathbf{R}^{n}$ with $M_{0}=\mathbf{C}_{0}$, and suppose that, for small enough $t, M_{t}$ can be expressed as the graph, taken off $\mathbf{C}_{0}$ over a domain $\Omega_{t}$, of a $C^{2}\left(\Omega_{t}\right)$ function $v_{t}$. (Thus $M_{t}=\left\{x+v_{t}(x) \eta(x): x \in \Omega_{t}\right\}$, where $\eta$ is a smooth unit normal for $\mathbf{C}_{0}$.) Suppose also that $v_{t}$ depends in a $C^{1}$ fashion on $t$, and that the $\Omega_{t}$ engulf all of $\mathbf{C}_{0}$ as $t \downarrow 0$ in the sense that $\cup_{s>0} \cap_{0<t<s} \Omega_{t}=\mathbf{C}_{0}$. Let $v$ be the initial velocity given by $v=\left.\frac{\partial v_{t}}{\partial t}\right|_{t=0}$. Then $v$ satisfies the equation $L_{\mathbf{C}_{0}} v=0$ on all of $\mathbf{C}_{0}$.

There are two special cases of this general principle which are important for us here:

EXAMPLE 1. Here we assume that the family $\left\{M_{t}\right\}$ is a family of cones $\left\{\mathbf{C}_{t}\right\}$ obtained by rotations of the given cone $\mathbf{C}_{0}$. In this case the initial velocity $v$ is a function which is homogeneous of degree 1 in the variable $r=|x|$; that is $v=r \varphi(\omega)$, where $r=|x|$ and $\omega=|x|^{-1} x$. Notice that if $\nu=\left(\nu^{1}, \ldots, \nu^{n}\right)$ is a smooth unit normal for $\mathbf{C}_{0}$ then the space of all such solutions which are generated by rotations as described is a finite dimensional space spanned by the solutions

$$
\omega^{i} \nu^{j}-\omega^{j} \nu^{i}, 1 \leq i<j \leq n
$$


EXAMPLE 2. Here we assume that the family $\left\{M_{t}\right\}$ is a family of translations of the given cone $\mathbf{C}_{0}$. In this case the initial velocity $v$ is a function which is homogeneous of degree 0 in the variable $r=|x|$; that is $v=\varphi(\omega)$, where $\omega=|x|^{-1} x$. The space of all such solutions generated by translations is a finite dimensional space spanned by the solutions

$$
\nu^{i}, i=1, \ldots, n
$$

where the notation is as in Example 1 above.

We can now describe the restrictions needed on $\mathbf{C}_{0}$ for the main theorem. We in fact need the following conditions:

$$
\ddagger\left\{\begin{array}{l}
\text { (a) } \mathbf{C}_{0} \text { is strictly stable and strictly minimizing. } \\
\text { (b) The solutions of } L_{\mathbf{C}_{0}} v=0 \text { which are generated by rotations and } \\
\text { translations as in Examples } 1 \text {, } 2 \text { above are the only homogeneous de- } \\
\text { gree } 1,0 \text { solutions of } L_{\mathbf{C}_{0}} v=0 \\
\text { (c) No eigenvalue } \lambda_{j} \text { of }-L_{\Sigma} \text { has the form }-q(n-3-q) \text {, where } q \text { is an } \\
\text { integer satisfying } 1 \leq q<(n-3) / 2 .
\end{array}\right.
$$

Remarks. (1) For a discussion of the terminology in $\ddagger(\mathrm{a})$ here, see [HS].

(2) Notice that the cones $\{\lambda \omega: \omega \in \Sigma, \lambda>0\}$, where $\Sigma$ is one of the minimal submanifolds of the sphere which are expressible as the cartesian product of a $p$-dimensional sphere with a $q$-dimensional sphere, with $p, q \geq 1, p+q=n-2 \geq 7$, do satisfy all the hypotheses $\ddagger(\mathrm{a}),(\mathrm{b}),(\mathrm{c})$. (For condition $\ddagger(a)$ see the thesis of G. Lawlor [LG], and for $\ddagger$ (b) see the table 2.6 in $[\mathrm{SS}]$; the condition $\ddagger(\mathrm{c})$ is checked directly, since we know the spectrum explicitly for products of spheres.) The cone over $S^{3} \times S^{3}$ does not satisfy $\ddagger(c)$ - there is a constant eigenfunction corresponding to the eigenvalue $\lambda_{1}=-6=-q(n-3-q)$ with $q=2$ and $n=8$; the cone over $S^{2} \times S^{4}$ fails condition $\ddagger$ (c) for a similar reason. Thus all the codimension 1 minimizing cones which intersect $S^{n-1}$ in a product of spheres satisfy the conditions $\ddagger$, except for the examples which are isometric to the cones over $S^{3} \times S^{3}$ or $S^{2} \times S^{4}$.

More generally, all the examples of minimizing cones in the lists of Lawson [L] and Ferus-Karcher [FK] do satisfy condition $\ddagger(a)$ (see the discussion in [SL2]), and there are no known counterexamples to condition $\ddagger$ (b) amongst 
the codimension 1 minimizing cones. However there are a number of counterexamples to condition $\ddagger(\mathrm{c})$, which were pointed out to me by B. Solomon. Solomon [SB1, 2] has explicitly computed the spectrum for $\Sigma=\mathbf{C}_{0} \cap S^{n-1}$ in case $\Sigma$ is a cubic isoparametric hypersurface. Two of these cubic examples correspond to cones $\mathbf{C}_{0}$, of dimensions 13 and 25 , which are are minimizing and satisfy $\ddagger(a),(b)$. (The remainder correspond to cones which are not minimizing.) The 13-dimensional minimizing example (in $\mathbf{R}^{14}$ ), does not satisfy $\ddagger(\mathrm{c})$, because the constant eigenfunction corresponds to eigenvalue $\lambda_{1}=-24$, and hence condition $\ddagger(\mathrm{c})$ is violated with $q=3$; the remaining example (which has dimension 25) does satisfy all the conditions $\ddagger$. There are also four examples of quartic isoparametric minimizing cones for which condition $\ddagger$ (c) fails with $q=6$ and one with $q=4$; in each case the eigenvalue involved is the first eigenvalue $\lambda_{1}$, which has constant eigenfunction. The remaining (infinitely many) examples of quartic isoparametric minimizing cones do satisfy $\ddagger$ (c). I am grateful to Bruce Solomon for acquainting me with these facts.

In all that follows, $\mathcal{M}$ will denote a "multiplicity one class" of $n$-dimensional embedded minimal submanifolds of $\mathbf{R}^{n+1}$, in the sense of [SL5]; thus each $M \in \mathcal{M}$ is an embedded hypersurface in some open $U_{M} \subset \mathbf{R}^{n+1}, \mathcal{H}^{n}(M \cap$ $K)<\infty$ for each compact $K \subset U_{M}$, and the class is closed under appropriate homotheties, rigid motions, and weak limits. More precisely, we assume:

1.1. (a) $M \in \mathcal{M} \Rightarrow q \circ \eta_{X, \rho} M \in \mathcal{M}$ and $q \circ \eta_{X, \rho} U_{M}=U_{q \circ \eta_{X, \rho} M}$ for each $X \in U_{M}$, each $\rho>0$, and for each orthogonal transformation $q$ of $\mathbf{R}^{n+1}$.

1.1. (b) If $\left\{M_{j}\right\} \subset \mathcal{M}$, if $U \subset \mathbf{R}^{n+1}$ with $U \subset U_{M_{j}}$ for all sufficiently large $j$ and if $\sup _{j \geq 1} \mathcal{H}^{n}\left(M_{j} \cap K\right)<\infty$ for each compact $K \subset U$, then there is a subsequence $M_{j^{\prime}}$ and an $M \in \mathcal{M}$ such that $U_{M_{j^{\prime}}} \supset U$ and $M_{j^{\prime}} \rightarrow M$ in $U$ in the sense that $\int_{M_{j^{\prime}}} f(X) d \mathcal{H}^{n}(X) \rightarrow \int_{M} f(X) d \mathcal{H}^{n}(X)$ for any fixed continuous $f: \mathbf{R}^{n+1} \rightarrow \mathbf{R}$ with compact support in $U$.

We also need here the additional property that each $M \in \mathcal{M}$ is an "oriented boundary" in $U_{M}$ in the sense that:

1.1. (c) Each $M \in \mathcal{M}$ is oriented and the multiplicity one current $\llbracket M \rrbracket$ associated with $M$ (obtained by integration of $n$-forms with compact support 
in $U_{M}$ over the hypersurface $M$ ) satisfies $\llbracket M \rrbracket=\partial \llbracket W_{M} \rrbracket$ in $U_{M}$ for some Lebesgue measurable subset $W_{M} \subset U_{M}$.

(Notice that (b) above is a strong restriction, in that it precludes, in particular, the possibility of getting varifolds with multiplicity greater than one on a set of positive measure as the varifold limit of a sequence $M_{j} \subset \mathcal{M}$ with each $U_{M_{j}} \supset U$ for some fixed open $U$; for this reason we refer to such a class as a multiplicity one class.)

By the discussion of [SL5], if $\mathcal{T}$ is the set of all $n$-dimensional currents $T$ which are boundaries of least area in some open subset $U_{T}$ of $\mathbf{R}^{n+1}$, then $\mathcal{M}=\{\operatorname{reg} T: T \in \mathcal{T}\}$ is such a muliplicity 1 class provided we take $U_{M}=U_{T}$ whenever $M=\operatorname{reg} T$ and $T \in \mathcal{T}$.

The following classes will be referred to frequently in what follows; here we use the notation

$$
Q_{\rho}=\left\{(x, y) \in \mathbf{R}^{n+1}: x \in B_{\rho},|y|<\rho\right\}
$$

and $\mathbf{C}=\mathbf{C}_{0} \times \mathbf{R} \in \mathcal{M}$, with $U_{\mathbf{C}}=\mathbf{R}^{n+1}$, is a cylinder with cross section $\mathbf{C}_{0}$ equal to an $(n-1)$-dimensional cone with vertex at 0 and with $\operatorname{sing} \mathbf{C}_{0}=\{0\}$.

1.2. Definition. $\mathcal{N}_{\epsilon}(\mathrm{C})$ is the set of all $M \in \mathcal{M}$ (where $\mathcal{M}$ is as above) such that $U_{M} \supset \bar{Q}_{1}, \int_{M \cap Q_{1}} \operatorname{dist}^{2}(X, \mathbf{C}) \leq \epsilon^{2}, \int_{{\mathrm{C} \cap Q_{1}}_{1}} \operatorname{dist}^{2}(X, M) \leq \epsilon^{2}$, and $\mathcal{H}^{n}\left(M \cap Q_{1}\right) \leq 1+\mathcal{H}^{n}\left(\mathbf{C} \cap Q_{1}\right)$

1.3. Remark. For given $\epsilon>0$ and any given $M \in \mathcal{M}$, if $X \in U_{M} \cap \bar{M} \backslash M$ is a (non-removable) isolated singular point for $M$, and if $\mathbf{C} \in \operatorname{Tan}_{X} M$, meaning $\eta_{X, \rho_{j}} M \rightarrow \mathbf{C}$ on $\mathbf{R}^{n+1}$ in the sense of $1.1(\mathrm{~b})$ for some sequence $\rho_{j} \downarrow 0$, then automatically $\eta_{X, \rho_{j}} M \in \mathcal{N}_{\epsilon}(\mathbf{C})$ for some sequence of $\rho_{j} \downarrow 0$; this follows directly from 1.1(b) and general fact that measure-theoretic convergence of minimal surfaces (as in 1.1(b)) implies Hausdorff distance sense convergence. (See e.g. [AW] or [SL3] for general discussion.)

We can now state the main theorem.

Theorem 1. Suppose $\mathbf{C}=\mathbf{C}_{0} \times \mathbf{R}$ with $\mathbf{C}_{0}$ satisfying $\ddagger(a),(b),(c)$ above. There is an $\epsilon=\epsilon\left(\mathbf{C}_{0}\right)>0$ such that if $M \in \mathcal{N}_{\epsilon}(\mathbf{C})$ with $\Theta_{M}(0) \geq \Theta_{\mathbf{C}}(0)$ then a rotation $\widetilde{\mathbf{C}}$ of $\mathbf{C}$ is the unique tangent cone of $M$ at 0 . That is, $\operatorname{Tan}_{0} M=\{\widetilde{\mathbf{C}}\}$. 
Remarks. (1) In the course of the proof, we give more precise information about how the convergence of $\eta_{0, \rho}$ to $\widetilde{\mathbf{C}}$ occurs as $\rho \downarrow 0$.

(2) With only minor modifications in the proof, it can be shown that the same holds if the part of $\ddagger$ (b) referring to homogeneous degree 1 solutions is replaced by the weaker requirement that all the homogeneous degree 1 solutions of $L_{\mathbf{C}_{0}} v=0$ are generated as in Example 1 above except that now the family $\mathbf{C}_{t}$ of minimal cones need not all be rotations of $\mathbf{C}_{0}$.

We shall need the following lemma, which is essentially exactly Lemma 2.6 from [SL5], except that we here work in cylinders $Q_{\rho}$ rather than in balls; the necessary modifications in the proof are very minor, so the proof of this modification is left to the reader.

1.4. Lemma. Suppose $\gamma, \beta, \tau \in(0,1)$ are arbitrary with $\tau \leq(1-\gamma) / 10$. There is $\epsilon_{0}=\epsilon_{0}(\mathbf{C}, \mathcal{M}, \gamma, \beta, \tau) \in(0,1]$ such that if $\mathbf{C}=\mathbf{C}_{0} \times \mathbf{R}^{m}$ is as in Remark 1.3 and if $M \in \mathcal{N}_{\epsilon_{0}}(\mathbf{C})$, then there is an open $U \subset \mathbf{C} \cap Q_{1}$ with the properties

$$
\begin{aligned}
(x, y) \in U \Rightarrow & (\widetilde{x}, y) \in U \text { whenever }(\widetilde{x}, y) \in \mathbf{C} \text { with }|\widetilde{x}|=|x| \\
& \left\{(x, y) \in \mathbf{C} \cap Q_{\gamma}:|x|>\tau\right\} \subset U
\end{aligned}
$$

and such that there is a $u \in C^{2}(U)$ with

$$
M \cap Q_{\gamma} \cap\{(x, y):|x|>\tau\} \subset \operatorname{graph} u \subset M, \quad \sup r^{-1}|u|+\sup |\nabla u| \leq \beta,
$$

and

$$
\int_{M \cap Q_{\gamma} \backslash \operatorname{graph} u} r^{2}+\int_{U \cap Q_{\gamma}} r^{2}|\nabla u|^{2} \leq C \int_{M \cap Q_{1}} d^{2}, \quad C=C(\mathbf{C}, \mathcal{M}, \gamma, \beta)
$$

note in particular that $C$ is independent of $\tau$. Here $r(x, y)=|x|, d(x, y)=$ $\operatorname{dist}((x, y), \mathbf{C}),(x, y) \in \mathbf{R}^{n} \times \mathbf{R}$.

Remarks. (1) Here

$$
\operatorname{graph} u=\{(x, y)+\eta(x) u(x, y):(x, y) \in U\}
$$

with $\eta(x)$ a smooth unit normal for the $\mathbf{C}_{0}$ which is normal to $e_{n+1}$. (Thus $\eta(x)$ is a unit normal for $\mathbf{C}$.) 
(2) By a simple modification of the of the argument in [SL5] used to establish the above bound, we can prove the more general inequality

$$
\int_{M \cap Q_{\gamma} \backslash \operatorname{graph} u} r^{2-\ell}+\int_{U \cap Q_{\gamma}} r^{2-\ell}|\nabla u|^{2} \leq C_{\ell} \int_{M \cap Q_{1}} \frac{d^{2}}{r^{\ell}}
$$

for each $\ell \geq 0$.

\section{2. $L^{2}$ Estimates}

The main result of this section is the following:

Theorem 2.1. Suppose $\mathbf{C}=\mathbf{C}_{0} \times \mathbf{R}$ is as described Remark 1.3, and suppose also that $\mathbf{C}_{0}$ is strictly stable and strictly minimizing (as in $\ddagger(a)$ ). There is $\epsilon_{0}=\epsilon_{0}(\mathbf{C})>0$ such that if $M \in \mathcal{N}_{\epsilon}(\mathbf{C})$ with $\epsilon \leq \epsilon_{0}$, then

$$
\int_{M \cap Q_{1 / 2}} r^{-2} d^{2}+\int_{U \cap Q_{1 / 2}}|\nabla u|^{2} \leq C \int_{M \cap Q_{1}} d^{2}
$$

where $C$ depends only on $\mathbf{C}$ and where $U, u$ are as in 1.4 .

Remark. This estimate does not require the fact that $\Theta_{M}^{n}(0) \geq \Theta_{\mathrm{C}}^{n}(0)$, nor does it use the hypotheses $\ddagger$ (b), (c).

The proof of Theorem 2.1, to be described at the end of this section, is a fairly direct consequence of the following two lemmas.

Lemma 2.2. If $\theta \in\left(\frac{3}{4}, 1\right)$ and $\rho \in\left(\frac{1}{2}, \frac{3}{4}\right)$, there is $\epsilon_{0}=\epsilon_{0}\left(\mathbf{C}_{0}, \theta\right) \in(0,1)$ such that if $\mathbf{C}=\mathbf{C}_{0} \times \mathbf{R}$ is as in Theorem 2.1 and if $M \in \mathcal{N}_{\epsilon}(\mathbf{C})$ for some $\epsilon \leq \epsilon_{0}$, then for all $\rho \in\left((1-\theta) \rho_{0}, \rho_{0}\right)$, with the exception of a set of Lebesgue measure $<(1-\theta) \rho / 4$,

$$
\begin{aligned}
& \int_{U \cap Q_{\rho}}|\nabla u|^{2}+\int_{M \cap Q_{\rho}} \frac{d^{2}}{r^{2}} \\
\leq & C_{1}\left(\left|M \cap Q_{\rho}\right|-\left|\mathbf{C} \cap Q_{\rho}\right|+\int_{M \cap Q_{\theta^{-1} \rho}}\left(\nu^{n+1}\right)^{2}\right)+C_{2}(1-\theta)^{-2} \int_{M \cap Q_{0-1} \rho} \frac{d^{2}}{r}
\end{aligned}
$$

where $Q_{\rho}=B_{\rho}^{n} \times(-\rho, \rho), \nu^{n+1}=e_{n+1} \cdot \nu$, and where $C_{j}=C_{j}(\mathbf{C}), j=1,2$. 
Proof. The proof involves a slicing argument, slicing orthogonal to the $e_{n+1}$ (i.e. orthogonal to the $y$ coordinate axis); we rely heavily on the theory of strictly minimizing cones introduced in [HS].

We first claim that, by virtue of the fact that $\mathbf{C}_{0}$ is strictly stable and strictly minimizing, there exist complete hypersurfaces $T_{ \pm} \subset E_{ \pm}$respectively having strictly negative mean curvature with respect to the unit normals pointing away from $\mathbf{C}_{0}$ and such that there is $R_{0}>0$ such that

$$
T_{ \pm} \backslash B_{R_{0}}=\left\{x+w_{ \pm} \eta(x): x \in \mathbf{C}_{0} \backslash V\right\}
$$

for some bounded open $V \subset \mathbf{C}_{0}$, where $w_{ \pm} \in C^{\infty}\left(\mathbf{C}_{0} \backslash V\right)$ and

$$
w_{ \pm} \sim r^{-\mu} \varphi_{1} \text { as } r \uparrow \infty
$$

in a sense to be made precise below. Here

$$
\mu=\frac{n-3}{2}-\sqrt{\left(\frac{n-3}{2}\right)^{2}+\lambda_{1}}
$$

note that by the strict stability of $\mathbf{C}_{0}$ we have $\mu>0$. (See [CHS] or [SJ].)

To check these claims, we use some of the results of [HS]. Let $E_{ \pm}$be the components of $\mathbf{R}^{n} \backslash \overline{\mathbf{C}}_{0}$. It is shown in [HS] that there are smooth complete embedded hypersurfaces $S_{ \pm} \subset E_{ \pm}$respectively such that for some $R_{0}>0$ we can write $S_{ \pm} \backslash B_{R_{0}}$ as graphs over $S_{ \pm}$in the sense that for some bounded domain $V \subset \mathbf{C}_{0}$

$$
S_{ \pm} \backslash B_{R_{0}}=\left\{x \pm w_{ \pm}(x) \eta(x): x \in \mathbf{C}_{0} \backslash V_{ \pm}\right\}
$$

where $\eta$ is the unit normal of $\mathbf{C}_{0}$ pointing into $E_{+}, U_{ \pm} \subset S_{ \pm}, S_{ \pm} \backslash U_{ \pm} \subset B_{R_{0}}$, and $s_{ \pm} \in C^{\infty}\left(S_{ \pm}\right)$with

$$
s_{ \pm}(x) \sim C r^{-\mu} \varphi_{1}(\omega) \text { as } r \uparrow \infty, r=|x|, \omega=|x|^{-1} x, C=\text { const. }
$$

where $\varphi_{1}$ is the first eigenfunction of the Jacobi field operator on the minimal submanifold $\Sigma=\mathbf{C}_{0} \cap S^{n-1}$ of $S^{n-1}$, and where the limits are in the $C_{*}^{2}$ sense-i.e.

$$
\left.\left.|| x\right|^{\mu} s_{ \pm}-\varphi\left(|x|^{-1} x\right)\right)\left.\right|_{C_{*}^{2}\left(S_{ \pm} \backslash B_{R}\right)} \leq C R^{-\alpha}, R \geq R_{0},
$$

where $|v|_{C_{*}^{2}}$ is the norm $\sup |v|+\sup (|x||\nabla v|)+\sup \left(|x|^{2}\left|\nabla^{2} v\right|\right)$. 
Now, as in [HS, Th.4.2], let $\mathcal{M}_{S_{ \pm}}$denote the minimal surface operator (i.e. Euler-Lagrange operator of the area functional) over $S_{ \pm}$respectively; thus if $w \in C^{2}\left(S_{ \pm}\right)$then $\mathcal{M}_{S_{ \pm}}(w)$ gives the mean curvature of the graph $\left\{x+w(x) \eta_{ \pm}(x): x \in S_{ \pm}\right\}$, where $\eta_{ \pm}$denote the unit normals of $S_{ \pm}$pointing away from $\mathbf{C}_{0}$. Then according to [HS, Th.4.2], for any non-negative $g_{ \pm} \in$ $C_{c}^{\infty}\left(S_{ \pm}\right)$and for any sufficiently small $\epsilon>0$, we can find smooth $\psi_{ \pm} \in C^{\infty}\left(S_{ \pm}\right)$ with $\mathcal{M}_{S_{ \pm}}\left(\psi_{ \pm}\right)=-\epsilon g_{ \pm}$on $S_{ \pm}$respectively and with $\psi_{ \pm}(x) \sim C|x|^{-\gamma} \varphi_{1}\left(|x|^{-1} x\right)$ for suitable constant $C>0$ as $|x| \uparrow \infty$, where $\varphi_{1}$ is as described above, and where $\gamma=(n-3) / 2+\sqrt{(n-3)^{2} / 4+\lambda_{1}}$, with $\lambda_{1}$ the (negative) first eigenvalue of the minimal submanifold $\Sigma$. On the other hand by the computation of [HS, pp.119-120] we have for any $\alpha>0$ and sufficiently small $\epsilon>0$ that $\mathcal{M}_{S_{ \pm}}\left(\epsilon r^{-\gamma-\alpha} \varphi_{1}(\omega)\right) \sim C \epsilon \rho^{-\gamma-\alpha-2} \varphi_{1}(\omega)$ for some constant $C>0$. Combining these facts, it is then easy to check that we can find positive $v_{ \pm} \in C^{\infty}\left(S_{ \pm}\right)$ (of the form $v_{ \pm}=\psi_{ \pm}-\delta r^{-\gamma-\alpha} \varphi_{1}$ outside the ball $B_{R_{0}}$ ) of arbitrarily small $C^{2}$ norm satisfying $\mathcal{M}_{S_{ \pm}}\left(v_{ \pm}\right)<0$ on $S_{ \pm}$and $v_{ \pm}(x) \sim C|x|^{-\gamma} \varphi_{1}\left(|x|^{-1} x\right)$ as $|x| \uparrow \infty$.

Now by definition $\mathcal{M}_{S_{ \pm}}\left(v_{ \pm}\right)<0$ means that we have strictly negative mean curvature for the graphs

$$
T_{ \pm}=\left\{x+v_{ \pm}(x) \eta_{ \pm}(x): x \in S_{ \pm}\right\}
$$

(which are of course embedded $C^{2}$ hypersurfaces if $v_{ \pm}$are chosen to have sufficiently small $C^{2}$ norms), provided the mean curvature is computed with respect to the unit normals $\eta_{ \pm}$for $S_{ \pm}$respectively. Also the construction described above ensures that for each $\epsilon>0$ we can select $v_{ \pm}$positive and such that

$$
\begin{aligned}
& \left|v_{ \pm}\right| C_{*}^{2}\left(S_{ \pm}\right) \leq \epsilon \\
& v_{ \pm}(x) \sim C|x|^{-\gamma} \varphi_{1}\left(|x|^{-1} x\right) \text { as }|x| \uparrow \infty
\end{aligned}
$$

where as above $|v|_{C_{*}^{2}}$ is the norm $\sup |v|+\sup (|x||\nabla v|)+\sup \left(|x|^{2}\left|\nabla^{2} v\right|\right)$ and where the error in the second statement holds in the sense that

$$
\|\left. x\right|^{\gamma} v_{ \pm}(x)-\left.C \varphi_{1}\left(|x|^{-1} x\right)\right|_{C_{*}^{2}\left(S_{ \pm} \backslash B_{R}\right)} \leq C R^{-\alpha}, R \text { sufficiently large }
$$

In view of the asymptotics (3) and (4), we deduce that there is $R_{0}>0$ such 
that

$$
T_{ \pm} \backslash B_{R_{0}}=\left\{x+w_{ \pm}(x) \eta(x): x \in \mathbf{C}_{0} \backslash V\right\}
$$

for some bounded open $V \subset \mathbf{C}_{0}$, where $w_{ \pm} \in C^{\infty}\left(\mathbf{C}_{0} \backslash V\right)$ and

$$
w_{ \pm}(r \omega) \sim C r^{-\mu} \varphi_{1}(\omega) \text { as } r \uparrow \infty
$$

where the error is again in the sense

$$
|||x|^{\mu} w_{ \pm}(x)-\left.C \varphi_{1}\left(|x|^{-1} x\right)\right|_{C_{*}^{2}\left(S_{ \pm} \backslash B_{R}\right)} \leq C R^{-\alpha}, R \text { sufficiently large }
$$

where $C, \alpha>0$.

Next we recall (from [HS]) that the hypersurfaces $S_{ \pm}$are "radial graphs" in the sense that each ray $\{\lambda \omega\}_{\lambda>0}$, where $\omega \in E_{ \pm}$, intersects $S_{ \pm}$transversely in precisely one point. In view of $(5),(6)$ it follows that the hypersurfaces $T_{ \pm}$ have the same radial graph property. We can therefore foliate all of $E_{+}$and $E_{-}$by the hypersurfaces $\left\{(\lambda) T_{ \pm}\right\}_{\lambda>0}$, where $(\lambda)$ is the homothety $x \mapsto \lambda x$. Take $X$ to be the unit normal vector field of this foliation; thus at points of $(\lambda) T_{ \pm}$we take $\left.X\right|_{x}$ to be \pm the unit normal vector of $(\lambda) T_{ \pm}$pointing away from $\mathbf{C}_{0} . \quad X$ extends in a $C^{1}$ fashion to $\mathbf{C}_{0}$ by taking $\left.X\right|_{x}=\eta(x)$ (the unit normal of $\mathbf{C}_{0}$ pointing into $\left.E_{+}\right)$in case $x \in \mathbf{C}_{0}$. The fact that the homotheties of $T_{ \pm}$have strictly negative mean curvature in $E_{ \pm}$means that $\pm \operatorname{div} X<0$ in $E_{ \pm}$.

Then we can define $\tilde{X}=\varphi\left(|x|^{-1} x\right) X$, with $\varphi$ a $C^{\infty}$ function on the sphere which is identically 1 on $\Sigma=\mathrm{C}_{0} \cap S^{n-1}$ and which takes only values in $\left(\frac{1}{2}, 1\right)$ on $S^{n-1} \backslash \Sigma$ (and which rapidly approaches 1 on approach to $\Sigma$ ) such that $\pm \operatorname{div} \tilde{X}<0$ on $E_{ \pm}$.

Before proceeding we need a couple more properties of the unit normal vector field $X$. Let $w_{ \pm}^{(\lambda)}(x)=\lambda w_{ \pm}\left(\lambda^{-1} x\right)$ for $\lambda>0$ and for $\lambda^{-1} x$ in the domain of $w_{ \pm}$respectively. Since $X$ is the normal vector field for the foliations of $E_{ \pm}$ given by the homotheties of $T_{ \pm}$, a point $\xi \in(\lambda) T_{ \pm}$will be in the graph of $w_{ \pm}^{(\lambda)}$ (i.e. $\xi$ can be written $\left.x+w_{ \pm}^{(\lambda)}(x) \eta(\omega)\right)$ provided

$$
\operatorname{dist}\left(|\xi|^{-1} \xi, \mathbf{C}_{0}\right) \leq \beta_{0}
$$


with $\beta_{0} \in(0,1)$ sufficiently small, depending on $\Sigma$ but not depending on $\lambda$. Notice that the restriction (7) is necessary in view of the fact that the representation (5) only applies outside $B_{R_{0}}$.

For a given hypersurface $L \subset \mathbf{R}^{n}$, we want now to examine the quantity $\left|X-\nu_{L}\right|^{2}$, where $X$ is the unit normal vector field associated with the foliations $\left\{\lambda T_{ \pm}\right\}_{\lambda>0}$ for $E_{ \pm}$and $\nu_{L}$ is an orienting smooth unit normal for $L$. For this purpose, first consider a neighbourhood of $L$ which can be represented as the graph of a function $\tilde{u} \in C^{1}(\Omega)\left(\Omega\right.$ a domain in $\left.\mathbf{C}_{0}\right)$ such that

$$
|x|^{-1}|\widetilde{u}(x)| \leq \beta_{0} \text { in } \Omega,
$$

where we take $\beta_{0}$ (depending on $\Sigma$ ) to be small enough so that (7) holds and also so that the nearest point projection of $\left\{x \in \mathbf{R}^{n} \backslash\{0\}: \operatorname{dist}\left(|x|^{-1} x, \mathbf{C}_{0}\right) \leq\right.$ $\left.\beta_{0}\right\}$ is well-defined and smooth.

At a point $\xi=x+\widetilde{u}(x) \eta(\omega) \in$ graph $\tilde{u}$, the tangent plane of $L$ is spanned by the vectors

$$
\left\{\tau_{i}+A_{\omega}\left(\tau_{i}\right) r^{-1} w^{(\lambda)}(x)+\nabla_{\tau_{i}} w^{(\lambda)}(x)\right\}
$$

where $A$ is the second fundamental form of $\Sigma$ at the point $|x|^{-1} x \in \Sigma$ and $\tau_{i}$ is an orthonormal basis for the tangent space of $\Sigma$ at the point $|x|^{-1} x$. So assume that $\widetilde{u} \in C^{1}(\Omega)$ is as in (8), let $x \in \Omega$ such that $\widetilde{u}(x) \neq 0$, and assume that $\lambda(x)>0$ is such that $w^{(\lambda)}(x)=\widetilde{u}(x)$, where $w^{(\lambda)}=w_{ \pm}^{(\lambda)}$ according as $\pm \widetilde{u}(x)>0$. It is then easily checked that

$$
\left.C^{-1}\left|\nabla w^{(\lambda)}(x)-\nabla \widetilde{u}(x)\right|^{2}\right|_{\lambda=\lambda(x)} \leq\left|X-\nu_{L}\right|^{2} \leq\left. C\left|\nabla w^{(\lambda)}(x)-\nabla \widetilde{u}(x)\right|^{2}\right|_{\lambda=\lambda(x)}
$$

where $\nu_{L}, X$ denote the unit normals of $L=\operatorname{graph} \widetilde{u}$ and graph $\left.w^{(\lambda)}\right|_{\lambda=\lambda(x)}$ at the point $x+\widetilde{u}(x) \eta(\omega)$. Since $w^{(\lambda)}(x)=\lambda w\left(\lambda^{-1} x\right)$ and since, at each point $x$, $\lambda(x)$ is chosen such that $w^{(\lambda(x))}(x)=\widetilde{u}(x)$, we see by direct calculation that

$$
\left|\nabla w^{(\lambda)}-\nabla \widetilde{u}\right|_{\lambda=\lambda(x)}^{2}=\left.\left(w\left(\lambda^{-1} x\right)^{2}\left|\nabla\left(\frac{\widetilde{u}(x)}{w\left(\lambda^{-1} x\right)}\right)\right|^{2}\right)\right|_{\lambda=\lambda(x)},
$$

(We emphasize that the gradients here are first computed for fixed $\lambda$, and then $\lambda$ is set equal to $\lambda(x)$.) Notice that by (6) we have that

$$
C^{-1}\left(\frac{|\widetilde{u}(x)|}{|x|}\right)^{1 /(1+\mu)} \leq \frac{\lambda(x)}{|x|} \leq C\left(\frac{|\widetilde{u}(x)|}{|x|}\right)^{1 /(1+\mu)}
$$


provided that $\frac{|\widetilde{u}(x)|}{|x|} \leq \beta_{0}$, where $C$ depends only on $\Sigma$, because (6) gives $C^{-1}|x|^{-\mu} \lambda^{\mu} \leq w^{ \pm}\left(\lambda^{-1} x\right) \leq C|x|^{-\mu} \lambda^{\mu}$ for $\lambda /|x| \leq \beta_{0}$.

Also, again using (6), we have

$$
\left|\frac{|x|^{\mu} w\left(\lambda^{-1} x\right)}{\lambda^{\mu} \varphi_{1}(\omega)}-1\right| \leq\left(\frac{\lambda}{|x|}\right)^{\alpha}, \quad\left|\nabla\left(\frac{w\left(\lambda^{-1} x\right)}{|x|^{-\mu} \varphi_{1}(\omega)}\right)\right| \leq C|x|^{-1}\left(\frac{\lambda}{|x|}\right)^{\alpha}
$$

for $\lambda^{-1}|x| \geq 1$. Then

$$
\begin{aligned}
\left|\nabla\left(\frac{\tilde{u}(x)}{w\left(\lambda^{-1} x\right)}\right)\right| & \equiv\left|\nabla\left(\frac{\tilde{u}(x)}{|x|^{-\mu} \varphi_{1}(\omega)} \frac{|x|^{-\mu} \varphi_{1}}{w\left(\lambda^{-1} x\right)}\right)\right| \\
& \geq \lambda^{\mu}\left|\nabla\left(\frac{\widetilde{u}}{|x|^{-\mu} \varphi_{1}(\omega)}\right)\right|-\lambda^{\mu}|x|^{-1} \frac{|\widetilde{u}(x)|}{|x|^{-\mu} \varphi_{1}}\left(\frac{\lambda}{|x|}\right)^{\alpha}
\end{aligned}
$$

and hence (using (12) again)

$$
\begin{aligned}
\left(w\left(\lambda^{-1} x\right)\left|\nabla\left(\frac{\tilde{u}(x)}{w\left(\lambda^{-1} x\right)}\right)\right|\right)^{2} & \\
& \geq C^{-1}\left(|x|^{-\mu}\left|\nabla\left(\frac{\widetilde{u}(x)}{|x|^{-\mu} \varphi_{1}(\omega)}\right)\right|\right)^{2}-C\left(\frac{|\widetilde{u}(x)|}{|x|}\right)^{2}\left(\frac{\lambda}{|x|}\right)^{2 \alpha}
\end{aligned}
$$

provided only $|x|^{-1}|\widetilde{u}(x)| \leq \beta_{0}$. Notice that by combining (9)-(13) we get

$$
\left|X-\nu_{L}\right|^{2} \geq C^{-1}\left(|x|^{-2 \mu}\left|\nabla \frac{\widetilde{u}(x)}{|x|^{-\mu} \varphi_{1}}\right|^{2}\right)-C\left(\frac{|\widetilde{u}(x)|}{|x|}\right)^{2+2 \alpha}
$$

for $|x|^{-1}|\widetilde{u}(x)| \leq \beta_{0}$. Also, the point $\xi=x+\widetilde{u}(x) \eta(\omega) \in L$ satisfies $|\xi|^{2}=$ $|x|^{2}+(\widetilde{u})^{2}$, and furthermore $\tilde{u}(x)=d(\xi)$, where $d(\xi)=\operatorname{dist}\left(\xi, \mathbf{C}_{0}\right)$, and hence it follows that, with $\nabla^{L}$ denoting the tangential gradient on $L$,

$$
\left|\nabla\left(\frac{\widetilde{u}}{|x|^{-\mu} \varphi_{1}(\omega)}\right)\right| \geq C^{-1}\left|\nabla_{\xi}^{L}\left(\frac{d(\xi)}{|\xi|^{-\mu} \varphi_{1}(\omega)}\right)\right|-C\left(\frac{d(\xi)}{|\xi|^{-\mu} \varphi_{1}(\omega)}\right)^{2}
$$

at all points $\xi$ of $L$ where (7) holds and where the unit normal satisfies $\nu_{L}(\xi)$. $\eta(\omega) \geq \frac{1}{2}$. (Notice that $\nu_{L} \cdot \eta(\omega) \geq \frac{1}{2}$ guarantees $|\nabla \widetilde{u}| \leq C$ with $C$ depending only on $\Sigma$; also, here $\omega$ is $|x|^{-1} x$, with $x$ the unique nearest point projection of $\xi$ onto $\mathbf{C}_{0}$.) Then by combining (14) and (15) we finally conclude

$$
\left|X(x)-\nu_{L}(x)\right|^{2} \geq C^{-1}\left(|x|^{-2 \mu}\left|\nabla^{L} \frac{d(x)}{|x|^{-\mu} \varphi_{1}(\omega)}\right|^{2}\right)-C\left(\frac{d(x)}{|x|}\right)^{2+2 \alpha}
$$

at all points of $L$ where $|x|^{-1} d(x) \leq \beta_{0}$, where $\alpha \in(0,1)$ depends only on $\Sigma$. (Actually the above argument established this at points where the additional 
fact $\nu_{L}(x) \cdot \eta(\omega) \geq \frac{1}{2}$ is true; on the other hand at all points of $L$ where $|x|^{-1} d(x) \leq \beta_{0}$ and $\nu_{L}(x) \cdot \eta(\omega) \geq \frac{1}{2}$ the inequality (16) is trivially true, because $\left|X-\nu_{L}\right| \geq \frac{1}{2}$ at such points-assuming again that $\beta_{0}$ is sufficiently small depending only on $\Sigma$.) We emphasize that (16) is a general inequality which holds for an arbitrary smooth hypersurface $L \subset \mathbf{R}^{n}$ in the region $|x|^{-1} d(x) \leq$ $\beta_{0}$, and the constant $C$ depends only on $\Sigma$.

Now we are going to make use of these properties to get information about the slices $M^{(y)}=\left\{x \in \mathbf{R}^{n}:(x, y) \in M\right\}$. First take $\rho \in\left(\frac{1}{2}, 1\right)$ and take $L$ to be any $(n-1)$-dimensional integer multiplicity current (or any oriented smooth hypersurface of finite $\mathcal{H}^{n-1}$-measure (not necessarily stationary) contained in $B_{\theta^{-1}} \rho$, with $\mathcal{H}^{n}\left(\operatorname{sing} L \cap B_{\theta^{-1}} \rho\right)=0$, with $\partial L=\partial\left(\mathbf{C}_{0}\left\llcorner B_{\theta^{-1}} \rho\right) \equiv \Sigma_{\theta^{-1} \rho} \equiv\{x \in\right.$ $\left.\mathbf{C}_{0}:|x|=\theta^{-1} \rho\right\}$ and with $L$ being an oriented boundary in $B_{\theta^{-1} \rho}$ in the sense $L=\partial \llbracket V_{L} \rrbracket$ in $B_{\theta^{-1} \rho}$, where $V_{L}$ is a Lebesgue measurable subset of $B_{\theta^{-1}} \rho$. Then we can write

$$
L-\left(\mathbf{C}_{0}\left\llcorner B_{\theta^{-1} \rho}\right)=\partial R\right.
$$

with $R$ an $n$-dimensional integral current with support in $B_{\theta^{-1} \rho}$ and with positive orientation in $E_{+}$and negative orientation in $E_{-}$. Thus if we take the form $\psi=\sum_{i=1}^{n}(-1)^{i-1} X_{i} d x^{1} \wedge \cdots \widehat{d x^{i}} \cdots \wedge d x^{n}$ (so that $d \psi=(\operatorname{div} \tilde{X}) d x^{1} \wedge$ $\left.\cdots \wedge d x^{n}\right)$, then, since $\pm \operatorname{div} \tilde{X} \leq 0$ in $E_{ \pm}$, we have

$$
\int_{L}\left\langle\tilde{X}, \nu_{L}\right\rangle \geq \int_{\mathrm{C}_{0}\left\llcorner B_{\theta^{-1} \rho}\right.}\langle\tilde{X}, \eta\rangle=\left|\mathbf{C}_{0} \cap B_{\theta^{-1} \rho}\right| .
$$

Since $\tilde{X}=\varphi X\left(\right.$ and hence $\left.\nu_{L} \cdot \tilde{X}=\varphi\left(1-\frac{1}{2}\left|X-\nu_{L}\right|^{2}\right)\right)$ we then conclude that (since $\frac{1}{2} \leq \varphi \leq 1$ )

$$
\int_{L}(1-\varphi)+\frac{1}{4} \int_{L}\left|\nu_{L}-X\right|^{2} \leq|L|-\left|\mathbf{C}_{0} \cap B_{\theta^{-1} \rho}\right| .
$$

For any $\delta \in\left(0, \beta_{0}\right)\left(\beta_{0}\right.$ as in the above discussion), define

$$
L_{\delta}=L\left\llcorner\left\{x: \operatorname{dist}\left(|x|^{-1} x, \mathbf{C}_{0}\right)>\delta\right\},\right.
$$

Notice by virtue of (16), (17) (after dropping the first term on the left of (17)) we have

$$
\int_{L_{\delta} \cap B_{\rho}} r^{-2 \mu}\left|\nabla\left(\frac{d}{r^{-\mu} \varphi_{1}(\omega)}\right)\right|^{2} \leq C\left(|L|-\left|\mathbf{C}_{0} \cap B_{\theta^{-1} \rho}\right|\right)+C \delta^{2 \alpha} \int_{L_{\delta} \cap B_{p}} r^{-2} d^{2},
$$


where $C$ does not depend on $\delta$. Notice also that if we drop the second term on the left of (17) then we obtain

$$
\delta_{1}\left|L \backslash L_{\delta}\right| \leq C\left(|L|-\left|\mathbf{C}_{0} \cap B_{\theta^{-1} \rho}\right|\right)
$$

where $\delta_{1}$ depends on $\delta$.

We are going to apply (18) to a composite hypersurface $L$ which agrees with a slice $M^{(y)}=\left\{x \in \mathbf{R}^{n}:(x, y) \in M\right\}$ on $B_{\rho}$. We in fact construct $L$ as follows:

By our assumption that $\int_{M \cap Q_{1}} d^{2} \leq \epsilon$, we know from Lemma 1.4 that

$$
M \cap B_{1} \backslash U \subset\{x \in M:|x|<\delta(\epsilon)\}, \quad \delta(\epsilon) \downarrow 0 \text { as } \epsilon \downarrow 0 .
$$

For $\epsilon$ sufficiently small, we can thus define $\widetilde{M}^{(y)} \subset B_{1}$ by taking

$$
\left\{\begin{aligned}
\widetilde{M}^{(y)} \cap B_{\rho} & =M^{(y)} \cap B_{\rho} \equiv\left\{x \in B_{\rho}:(x, y) \in M\right\} \\
\widetilde{M}^{(y)} \backslash B_{\rho} & =\operatorname{graph} \widetilde{u}
\end{aligned}\right.
$$

where $v \in C^{2}\left(\bar{B}_{\theta^{-1} \rho} \backslash B_{\rho}\right)$ agrees with $u(\cdot, y)$ on $B_{\rho}$ and interpolates linearly (as a function of $|x|$ on a ray $x=\lambda \omega$ ) between $u(\rho \omega, y)$ at $|x|=\rho$ and zero at $|x|=\theta^{-1} \rho$. Now by standard elliptic estimates we have

$$
\begin{aligned}
& \sup _{\operatorname{Cn}\left\{\rho / 3<|x|<\frac{1}{2}\left(1+\theta^{-1}\right) \rho,|y|<\frac{1}{2}\left(1+\theta^{-1}\right) \rho\right\}}\left(|u|^{2}+|\nabla u|^{2}\right) \\
& \leq C(1-\theta)^{-2} \int_{C \cap\left\{\rho / 4<|x|<\rho,|y|<\theta^{-1} \rho\right\}}\left(u^{2}+|\nabla u|^{2}\right),
\end{aligned}
$$

and so (18) gives, with $v$ defined as in (19),

$$
\begin{gathered}
\int_{\mathrm{C}_{0} \cap\left\{x: \rho / 3<|x|<\theta^{-1} \rho\right\}}\left(v^{2}+|\nabla v|^{2}\right) \\
\leq C \int_{\operatorname{C} \cap\left\{\rho / 4<|x|<\theta^{-1} \rho,|y|<\theta^{-1} \rho\right\}}\left(u^{2}+|\nabla u|^{2}\right) .
\end{gathered}
$$

Notice in particular that it then follows directly (using the appropriate representation of the area functional over $\mathbf{C}_{0}$ to represent the area of $\widetilde{M}^{(y)}$ in the 
annular region $\left.\left\{x: \rho<|x|<\theta^{-1} \rho\right\}\right)$ that

$$
\begin{aligned}
& \left|\widetilde{M}^{(y)} \cap\left\{x: \rho<|x|<\theta^{-1} \rho\right\}\right|-\left|\mathbf{C} \cap\left\{x: \rho<|x|<\theta^{-1} \rho\right\}\right| \\
\leq C & \int_{\mathbf{C}_{0} \cap Q_{\theta^{-1}} \cap\{\rho / 4<|x|\}}\left(u^{2}+|\nabla u|^{2}\right)
\end{aligned}
$$

for any $y \in\left(0, \frac{1}{2}\left(1+\theta^{-1}\right) \rho\right)$. Then, in view of $(21),(18)$ implies

$$
\begin{aligned}
& \int_{M_{\delta}^{(y)} \cap B_{\rho}} r^{-2 \mu}\left|\nabla^{\prime}\left(\frac{d}{r^{-\mu} \varphi_{1}(\omega)}\right)\right|^{2} \\
\leq & C\left(\left|M^{(y)} \cap B_{\rho}\right|-\left|\mathbf{C}_{0} \cap B_{\rho}\right|\right) \\
& +C\left(\delta^{2 \alpha} \int_{M_{\delta}^{(y)} \cap B_{\rho}} r^{-2} u^{2}+\int_{U \cap\left\{\rho / 4<|x|<\theta^{-1} \rho,|y|<\theta^{-1} \rho\right\}}\left(u^{2}+|\nabla u|^{2}\right)\right)
\end{aligned}
$$

with $C$ independent of $\delta$, where

$$
M_{\delta}^{(y)}=\left\{x \in \mathbf{R}^{n}:(x, y) \in M \text { and dist }\left(|x|^{-1} x, \mathbf{C}_{0}\right)>\delta\right\}
$$

and where $\nabla^{\prime}$ denotes gradient in the slice $U^{(y)}$. Now we integrate with respect to $y$ over the interval $(-\rho, \rho)$; this implies

$$
\begin{aligned}
& \int_{M \cap Q_{\rho} \backslash Y} r^{-2 \mu}\left|\nabla^{\prime}\left(\frac{d}{r^{-\mu} \varphi_{1}(\omega)}\right)\right|^{2} \\
\leq & C\left(\left|M \cap Q_{\rho}\right|-\left|\mathbf{C} \cap Q_{\rho}\right|\right) \\
& +C \delta^{2 \alpha} \int_{M \cap\left(B_{0^{-1}} \times(-\rho, \rho)\right) \backslash Y} r^{-2} d^{2}+C \int_{\mathrm{C}_{0} \cap Q_{0^{-1}} \cap \cap\{\rho / 4<|x|\}}\left(u^{2}+|\nabla u|^{2}\right),
\end{aligned}
$$

where $Y=\left\{(x, y) \in M: \operatorname{dist}\left(|x|^{-1} x, \mathbf{C}_{0}\right) \leq \delta\right\}$. Since $\nabla^{\prime} f(x, y)$ just means 
$\nabla_{x} f(x, y)$, this gives

$$
\begin{aligned}
& \int_{Q_{\rho} \cap M \backslash Y} r^{-2 \mu}\left|\nabla\left(\frac{d}{r^{-\mu} \varphi_{1}(\omega)}\right)\right|^{2} \\
\leq & C\left(\left|M \cap Q_{\rho}\right|-\left|\mathbf{C} \cap Q_{\rho}\right|\right)+C \delta^{2 \alpha} \int_{M \cap\left(B_{0-1} \times(-\rho, \rho)\right) \backslash Y} r^{-2} d^{2} \\
+ & C\left(\int_{Q_{0^{-1}} \cap}\left(u_{\{\rho / 4<|x|\}}\left(u^{2}+|\nabla u|^{2}\right)+\int_{Q_{\rho} \cap U} d_{y}^{2}\right),\right.
\end{aligned}
$$

where $d_{y}=e_{n+1} \cdot \nabla^{M} d \equiv \nu^{n+1} \sum_{j=1}^{n} \nu^{j} D_{j} d$ by virtue of the fact that $d$ is independent of the $y$ variable.

Next we claim that for any $\kappa<(n-3) / 2$ and any Lipschitz $v$ on $M$

$$
\int_{M \cap Q_{\rho}} r^{-2} v^{2} \leq C\left((1-\theta)^{-1} \int_{M \cap Q_{\theta^{-1} \rho}} r^{-1} v^{2}+\int_{M \cap\left(B_{0-1} \times(-\rho, \rho)\right)} r^{-2 \kappa}\left|\nabla\left(r^{\kappa} v\right)\right|^{2}\right),
$$

for all $\rho \in\left((1-\theta) \rho_{0}, \rho_{0}\right)$ with the exception of a set of measure $(1-\theta) \rho_{0} / 4$, where $C$ depends only on $\kappa$ and $\Sigma$. This is proved by making a special choice of $\Phi$ in the first variation identity

$$
\int_{M} \operatorname{div} \Phi=0
$$

valid for any $\Phi=\left(\Phi^{1}, \ldots, \Phi^{n+1}\right): \mathbf{R}^{n+1} \rightarrow \mathbf{R}^{n+1}$ with $\Phi^{j} \in C_{c}^{\infty}\left(Q_{1}\right)$. In this formula we take $\Phi(x, y)=\zeta(y) \varphi(r) v^{2}(x, y)(x, 0)$, with $r=|x| \varphi, \zeta \in C_{c}^{\infty}(\mathbf{R})$ with supports $\subset\left(-\theta^{-1} \rho, \theta^{-1} \rho\right)$ and $v \in C^{\infty}\left(B_{2}\right)$, and $\varphi \equiv 1$ near 0 . This leads by direct computation (Cf. the proof of inequality 2.5 of [SL5]) to the identity

$$
\begin{gathered}
\int_{M}\left(\left((n-1)+\left(\nu^{n+1}\right)^{2}\right) v^{2} \zeta(y) \varphi(r)+v^{2} \zeta(y) \sum_{i, j=1}^{n} x^{i} x^{j} g^{i j} r^{-1} \varphi^{\prime}(r)\right) \\
=\int_{M}\left(v^{2} \sum_{i=1}^{n} \nu^{i} x^{i} \varphi(r) \nu^{n+1} \zeta^{\prime}(y)-2 v \zeta(y) \varphi(r) \sum_{i=1}^{n} \sum_{j=1}^{n+1} x^{i} g^{i j} v_{j}\right) .
\end{gathered}
$$

Since $\sum_{i, j=1}^{n} g^{i j} x^{i} x^{j} \leq r^{2}$, this gives

$$
\begin{aligned}
& \int_{M}\left((n-1)+\left(\nu^{n+1}\right)^{2}\right) \varphi(r) \zeta(y) v^{2} \\
\leq & \int_{M}\left(r\left|\varphi^{\prime}(r)\right| \zeta(y) v^{2}+\left|\sum_{i=1}^{n} x^{i} \nu^{i}\right|\left|\nu^{n+1}\right| \varphi(r)\left|\zeta^{\prime}(y)\right| v^{2}+2 r v|\nabla v| \varphi(r) \zeta(y)\right),
\end{aligned}
$$


and hence

$$
\begin{aligned}
& (n-1) \int_{M} \varphi(r) \zeta(y) v^{2} d \mu \\
& \quad \leq \int_{M}\left(r\left|\varphi^{\prime}(r)\right| \zeta(y) v^{2}+2 r \varphi(r) \zeta(y) v|\nabla v|+r v^{2}\left|\zeta^{\prime}(y)\right| \varphi(r)\right) d \mu
\end{aligned}
$$

Now let $\zeta$ approximate the characteristic function of the interval $(-\rho, \rho)$, select $\varphi(r)=r^{-2 \kappa-2} \eta^{2}(r)$, where $\eta$ is a cut-off function which is 1 in $B_{\rho}$ and zero outside $B_{\theta^{-1} \rho}$, and replace $v$ by $r^{\kappa} v$. Then the previous inequality gives

$$
\begin{aligned}
(n-3-2 \kappa) & \int_{M \cap\left(B_{0-1} \times(-\rho, \rho)\right)} r^{-2} v^{2} \\
\leq & \int_{M \cap\left(B_{0-1} \times(\{-\rho\} \cup\{\rho\})\right)} r^{-1} v^{2} \eta^{2} \\
& +C\left(\int_{M \cap\left(B_{0^{-1} \rho} \times(-\rho, \rho)\right)} r^{-1-\kappa} v\left|\nabla\left(r^{\kappa} v\right)\right| \eta^{2}+\eta\left|\eta^{\prime}\right| r^{-1} v^{2}\right) .
\end{aligned}
$$

Now by assumption $n-3-2 \kappa>0$, and by virtue of the coarea formula we know that

$$
\int_{M \cap B_{\theta-1} \times(\{-\rho\} \cup\{\rho\})} r^{-1} v^{2} \eta^{2} \leq C(1-\theta)^{-1} \int_{M \cap Q_{\theta^{-1}}} r^{-1} v^{2} \eta^{2}
$$

with the exception of a set of $\rho \in\left((1-\theta) \rho_{0}, \rho_{0}\right)$ of measure $\leq(1-\theta) \rho_{0} / 4$, and so this implies

$$
\begin{aligned}
& \int_{M \cap\left(B_{\theta^{-1}} \times(-\rho, \rho)\right)} r^{-2} v^{2} \\
\leq & \int_{M \cap B_{\theta^{-1}} \times(\{-\rho\} \cup\{\rho\})} r^{-1} v^{2} \eta^{2}+C\left(\int_{M \cap Q_{0^{-1}} \rho} r^{-1-\kappa} v\left|\nabla\left(r^{\kappa} v\right)\right| \eta^{2}+\eta\left|\eta^{\prime}\right| r^{-1} v^{2}\right),
\end{aligned}
$$

with $C$ depending only on $\kappa$ and $\Sigma$. After an application of the Cauchy inequality we then obtain the required inequality (23).

Next, let $d_{\delta}$ be defined by

$$
d_{\delta}(X)= \begin{cases}\operatorname{dist}\left(x, \mathbf{C}_{0}\right), & \text { if }|x|^{-1} \operatorname{dist}\left(x, \mathbf{C}_{0}\right)<\delta \\ \delta|x|, & \text { if }|x|^{-1} \operatorname{dist}\left(x, \mathbf{C}_{0}\right) \geq \delta\end{cases}
$$


Using (23) with $\kappa=\mu$ and $v=d_{\delta} / \varphi_{1}(\omega)$, we obtain (since $d_{\delta}=\delta|x|$ on $Y$ )

$$
\begin{aligned}
& \int_{M \backslash Y \cap B_{o_{\rho} \times(-\rho, \rho)}} r^{-2} d^{2} \\
\leq C & \int_{M \cap Q_{\rho}} d^{2}+C \delta^{2 \alpha}\left|Y \cap Q_{\rho}\right|+\int_{M \cap Q_{\rho} \backslash Y} r^{-1} d^{2} \\
+ & C \int_{M \cap Q_{\rho} \backslash Y} r^{-2 \mu}\left|\nabla\left(\varphi_{1}(\omega)^{-1} r^{\mu} d(x)\right)\right|^{2} .
\end{aligned}
$$

On the other hand by $(18)^{\prime}$ we have

$$
\delta_{1}\left|Y^{(y)} \cap B_{\rho}\right| \leq\left|M^{(y)} \cap B_{\rho}\right|-\left|\mathbf{C}_{0} \cap B_{\rho}\right|+C \int_{Q_{0^{-1}} \cap\{|x|>\rho / 4\}}\left(u^{2}+|\nabla u|^{2}\right),
$$

and, integrating this inequality with respect to $y$, we get

$$
\delta_{1}\left|Y \cap Q_{\rho}\right| \leq\left|M \cap Q_{\rho}\right|-\left|\mathbf{C}_{0} \cap Q_{\rho}\right|+C \int_{\mathbf{C}_{0} \cap Q_{\theta^{-1}} \cap\{|x|>\rho / 4\}}\left(u^{2}+|\nabla u|^{2}\right) .
$$

Combining (22), (26) and (27) and using (1.5) with $\ell=2$ (and keeping in mind that $d \leq r)$, we then finally get the required inequality.

Lemma 2.3. If $\theta \in\left(\frac{3}{4}, 1\right)$ and $\rho_{0} \in\left(\frac{1}{2}, 1\right)$, there is $\epsilon_{0}=\epsilon_{0}\left(\mathbf{C}_{0}\right)>0$ such that if $\mathbf{C}=\mathbf{C}_{0} \times \mathbf{R}$ is as in Theorem 2.1 and if $M \in \mathcal{N}_{\epsilon}(\mathbf{C})$ with $\epsilon \leq \epsilon_{0}$, then

$\int_{M \cap Q_{\rho}}\left(\nu^{n+1}\right)^{2}+\left|M \cap Q_{\rho}\right|-\left|\mathbf{C} \cap Q_{\rho}\right| \leq C(1-\theta)^{-1} \int_{M \cap Q_{0^{-1}},} r^{-1} d^{2}, \quad C=C\left(\mathbf{C}^{(0)}\right)$, for all $\rho \in\left((1-\theta) \rho_{0}, \rho_{0}\right)$ with the exception of a set of 1-dimensional Lebesgue measure less than $(1-\theta) \rho_{0} / 4$.

Proof. Take any $\rho \in\left(\rho_{0} / 2, \rho_{0}\right)$. We begin by letting $\zeta$ and $\varphi$ approximate the characteristic function of the intervals $[-\rho, \rho]$ and $(-\infty, \rho]$ respectively in the identity (24) of the proof of Lemma 2.2 with $v \equiv 1$. Since $\left|\nu^{n+1} \sum_{i=1}^{n} x^{i} \nu^{i}\right| \leq$ $r^{-2} u^{2}+|\nabla u|^{2}$ on $G=\operatorname{graph} u$, this gives

$$
\begin{aligned}
& \int_{M \cap Q_{\rho}}\left(\nu^{n+1}\right)^{2}+(n-1)\left|M \cap Q_{\rho}\right| \\
& \leq \rho\left|I_{\rho}\right|+C \int_{U \cap Q_{\rho}} r\left(|\nabla u|^{2}+r^{-2} u^{2}\right)+\int_{Z^{(\rho)} \cup Z^{(-\rho)}} r d \mathcal{H}^{n-1},
\end{aligned}
$$


where $I_{\rho}$ is the $(n-1)$-dimensional manifold

$$
I_{\rho}=M \cap\left(\left(\partial B_{\rho}\right) \times(-\rho, \rho)\right),
$$

where $Z^{(\sigma)}=(M \backslash G) \cap\left(\mathbf{R}^{n} \times\{\sigma\}\right)$, and where $G=$ graph $u$ with $U, u$ as in 1.4, 1.5 .

Now let $\Sigma_{\rho}=\left\{\rho \omega: \omega \in \mathbf{C}_{0} \cap S^{n-1}\right\}$. One readily checks (by computing the area functional for $I_{\rho}$ over the stationary manifold $\Sigma_{\rho}$ explicitly in terms of $u$ ), that

$$
\left|I_{\rho}\right|-\left|\Sigma_{\rho} \times(-\rho, \rho)\right| \leq\left. C \int_{\Sigma_{\rho} \times(-\rho, \rho)}\left(|\nabla u|^{2}+r^{-2} u^{2}\right)\right|_{r=\rho} .
$$

Thus we have (since $\left.\rho\left|\Sigma_{\rho} \times(-\rho, \rho)\right|=(n-1)\left|\mathbf{C} \cap Q_{\rho}\right|\right)$

$$
\begin{aligned}
\int_{M \cap Q_{\rho}}\left(\nu^{n+1}\right)^{2}+\mid M & \cap Q_{\rho}|-| \mathbf{C} \cap Q_{\rho} \mid \\
& \leq C \int_{U \cap \partial Q_{\rho}} r\left(|\nabla u|^{2}+r^{-2} u^{2}\right)+C \int_{Z^{(\rho)} \cup Z^{(-\rho)}} r d \mathcal{H}^{n-1} .
\end{aligned}
$$

The proof is now completed by using the co-area formula in combination with (1); indeed the co-area formula enables us to assert that for all $\rho \in\left(\rho_{0} / 2, \rho_{0}\right)$, with the exception of a set of 1-dimensional Lebesgue measure less than (1$\theta) \rho_{0} / 2$,

$$
\begin{aligned}
\int_{U \cap \partial Q_{\rho}} r\left(|\nabla u|^{2}+r^{-2} u^{2}\right) & +\rho \int_{Z^{(\rho)} \cup Z^{(-\rho)}} r d \mathcal{H}^{n-1} \\
& \leq C \rho^{-1}\left(\int_{U \cap Q_{\theta^{-1} \rho}} r\left(|\nabla u|^{2}+r^{-2} u^{2}\right)+\int_{M \cap Q_{0-1} \backslash G} r\right),
\end{aligned}
$$

where $C=C(\theta, n)$ and $Z=M \backslash G$, and hence (1), together with 1.4 and 1.5 (with $\ell=1$ ) gives us the required inequality.

We can now give the proof of the main $L^{2}$ estimate 2.1:

Proof of Theorem 2.1. Notice first that by combining Lemma 2.2 and Lemma 2.3 we have a constant $\gamma$ such that for any $\theta \in\left(\frac{3}{4}, 1\right)$ and any $\rho_{0} \in\left(\frac{1}{2}, \frac{3}{4}\right)$

$$
\int_{M \cap Q_{\rho}} \frac{d^{2}}{r^{2}} \leq C(1-\theta)^{-\gamma} \int_{M \cap Q_{\theta^{-1} \rho}} \frac{d^{2}}{r}, \quad C=C(\Sigma), \gamma=\gamma(\Sigma)^{\prime},
$$


for some $\rho \in\left[(1-\theta) \rho_{0}, \rho_{0}\right]$. The constant $C$ does not depend on $\rho_{0}$ or $\theta$, and hence it is easy to check that this inequality then actually holds for every $\rho \in\left(\frac{1}{2}, \frac{3}{4}\right)$, with a new constant $C$ (still independent of $\theta$ ), and every $\theta \in\left(\frac{3}{4}, 1\right)$. By Hölder's inequality we then obtain

$$
I(\rho) \leq C(1-\theta)^{-q} \sqrt{I\left(\theta^{-1} \rho\right)} \sqrt{J\left(\theta^{-1} \rho\right)},
$$

where $I(\rho)=\int_{M \cap Q_{\rho}} r^{-2} d^{2}$ and $J(\rho)=\int_{M \cap Q_{\rho}} d^{2}$. Replacing $\rho$ by $\rho_{k}=\frac{1}{2}(1+$ $\theta) \rho\left(1-2^{-k}\right)$, so that $\rho_{k+1}=\theta_{k}^{-1} \rho_{k}$, where $\theta_{k}=\left(1-2^{-k}\right) /\left(1-2^{-k-1}\right)$, and iterating in (1) with $k=k_{0}, k_{0}+1, \ldots$, where $k_{0}$ is such that $1-2^{-k_{0}}=$ $2 \theta /(1+\theta)$, we finally conclude

$$
\int_{M \cap Q_{0 \rho}} \frac{d^{2}}{r^{2}} \leq C \int_{M \cap Q_{\rho}} d^{2}, \quad \rho \in\left(\frac{1}{2}, \frac{3}{4}\right) .
$$

Then the remainder of 2.1 follows directly from this and 1.5.

An important corollary of 2.1 is the following, which shows that the contribution to the integral $\rho^{-n-2} \int_{Q_{\rho} \cap M} d^{2}$ from points near the axis $\{0\} \times \mathbf{R}$ is "negligible" in a certain sense.

Corollary 2.3. With the notation and assumptions of 2.1, for each each $\sigma \in$ $(0,1)$

$$
\int_{M \cap Q_{1 / 2} \cap\{(x, y):|x| \leq \sigma\}} d^{2} \leq C \sigma^{2} \int_{M \cap Q_{1}} d^{2} .
$$

Proof. The proof is simply a matter of noting that the integral $\int_{M \cap Q_{1 / 2}} r^{-2} d^{2}$ appearing on the left of 2.1 is $\geq \sigma^{-2} \int_{M \cap Q_{1 / 2} \cap\{(x, y):|x| \leq \sigma\}} d^{2}$.

\section{Proof of Theorem 1}

As we mentioned in the introduction, the idea is to use the "blowup" method; in the context of unique tangent cone problems, the method of blowing up was first used by Almgren and Allard [AA] to discuss uniqueness of $\mathbf{C}$ in the case when $\mathbf{C}$ had an isolated singularity at 0 and multiplicity 1 elsewhere. An integrability condition (like the the part of condition $\ddagger$ (b) above referring to homogeneous degree one solutions) was needed in [AA], but no conditions like $\ddagger(\mathrm{a}),(\mathrm{c})$, nor like the part of $\ddagger$ (b) referring to homogeneous degree zero 
solutions. (Using a different method, the present author later showed in [SL1] that no integrability conditions are needed to handle the case when $\mathbf{C}$ has an isolated singularity at 0 and multiplicity 1 elsewhere.)

One of the key difficulties in using the blowup method in the context of non-isolated singular points is the necessity of obtaining sufficiently strong a-priori $L^{2}$ estimates; this was precisely the reason for the discussion in the previous section.

A very brief overall idea of the blowup method is as follows. We begin by assuming that the hypotheses of Theorem 1 hold with sequences $\epsilon_{k}, M_{k}$ in place of $\epsilon, M$ respectively, where $\epsilon_{k} \downarrow 0$, and let $M_{k}, U_{k}, u_{k}, d_{k}$ be the quantities corresponding to $M, U, u, d$ with $M$ replaced by $M_{k}$. Then we make an analysis of the behaviour of $\delta_{k}^{-1} d_{k}\left(\approx \delta_{k}^{-1} u_{k}\right.$ over the domain $\left.U_{k}\right), \delta_{k}=\left(\int_{M \cap B_{1}} d_{k}^{2}\right)^{1 / 2}$ as $k \uparrow \infty$. The weak limit $v$ of $\delta_{k}^{-1} u_{k}$ gives a smooth solution of the Jacobi field operator on $\mathbf{C}$, and the $L^{2}$ estimates of the previous section, together with the assumptions $\ddagger$, make it possible to precisely analyse $v$ and to then go back to recover information about the original sequence $u_{k}$. We now implement this procedure in detail.

Then let $\epsilon_{k}, M_{k}, U_{k}, u_{k}, d_{k}$ be sequences as above, and let $\delta_{k}=\sqrt{\int_{M_{k} \cap B_{1}} d_{k}^{2}}$, $v_{k}=\delta_{k}^{-1} u_{k}$. By virtue of the estimate 2.1 we have that

$$
\int_{U_{k} \cap B_{0}}\left(\left|\nabla v_{k}\right|^{2}+r^{-2} v_{k}^{2}\right) \leq C,
$$

for each $\theta \in(0,1)$ where $C$ depends on $\theta$ but not on $k$. Then by virtue of Rellich's Theorem we have that $v_{k}$ converges strongly in $L^{2}\left(B_{\theta} \backslash\{(x, y):|x| \geq\right.$ $\sigma\}$ ), for each $\sigma>0$ and for each $\theta \in(0,1)$, to $v \in W_{\text {loc }}^{1,2}\left(B_{1}\right)$, where

$$
\int_{\mathrm{C} \cap B_{0}}\left(|\nabla v|^{2}+r^{-2} v^{2}\right)<\infty
$$

Furthermore by 4.4 we have that

$$
\int_{U_{k} \cap B_{0}} R^{2-n}\left(\frac{\partial}{\partial R}\left(v_{k} / R\right)\right)^{2} \leq C
$$


where again $C$ depends on $\theta$ but not on $k$, and hence

$$
\int_{\mathbf{C} \cap B_{\theta}} R^{2-n}\left(\frac{\partial}{\partial R}(v / R)\right)^{2}<\infty .
$$

Now we need to analyse the function $v$ further; first note that since the functions $u_{k}$ satisfy the equation

$$
\mathcal{M}\left(u_{k}\right)=0 \text { on } \mathbf{C} \cap B_{1},
$$

and hence

$$
\frac{\partial^{2} u_{k}}{\partial y^{2}}+r^{2-n} \frac{\partial}{\partial r}\left(\frac{r^{n-2} \partial u_{k}}{\partial r}\right)+r^{-2} L_{\Sigma} u_{k}=R_{k},
$$

where $\left|R_{k}\right| \leq C \epsilon_{k} r^{-1}\left(r^{-1}\left|u_{k}\right|+\left|\nabla u_{k}\right|\right)$, and hence after multiplying through by $\delta_{k}^{-1}$ and taking limits (which exist in the space $W_{l o c}^{2, p} \forall p$ by the $L^{p}$ theory for elliptic equations), we conclude that $v$ also satisfies the Jacobi-field equation

$$
\frac{\partial^{2} v}{\partial y^{2}}+r^{2-n} \frac{\partial}{\partial r}\left(\frac{r^{n-2} \partial v}{\partial r}\right)+r^{-2} L_{\Sigma} v=0 \quad \text { on } B_{1} \backslash\{0\} \times \mathbf{R}
$$

We now proceed to analyse solutions of (7) which satisfy the conditions (2) and (4). To begin, take the $L^{2}(\Sigma)$ inner product with the $j^{\text {th }}$ eigenfunction $\varphi_{j}(\omega)$ of the operator $-L_{\Sigma}$, and note that this satisfies the equation

$$
\frac{\partial^{2}}{\partial y^{2}}\left\langle v, \varphi_{j}\right\rangle+\frac{1}{r^{n-2}} \frac{\partial}{\partial r}\left(r^{n-2} \frac{\partial}{\partial r}\left\langle v, \varphi_{j}\right\rangle\right)-\lambda_{j}\left\langle v, \varphi_{j}\right\rangle=0 .
$$

Now let $v_{j}(r, y)=r^{-\mu_{j}}\left\langle v, \varphi_{j}\right\rangle$, where $\mu_{j}=-(n-3) / 2+\sqrt{(n-3)^{2} / 4+\lambda_{j}}$. Then $v_{j}$ satisfies the equation

$$
\frac{1}{r^{1+\beta_{j}}} \frac{\partial}{\partial r}\left(r^{1+\beta_{j}} \frac{\partial v_{j}}{\partial r}\right)+\frac{\partial^{2} v_{j}}{\partial y^{2}}=0
$$

where $\beta_{j}=2 \sqrt{(n-3)^{2} / 4+\lambda_{j}}$. Also from (2) and (4) we obtain

$$
\int_{(0, \rho) \times(-\rho, \rho)}\left(\left|\nabla v_{j}\right|^{2}+r^{-2} v_{j}^{2}\right) r^{1+\beta_{j}} d r d y<\infty, \quad \rho<\frac{1}{\sqrt{2}},
$$

and

$$
\int_{(0, \rho) \times(-\rho, \rho)}\left(1+y^{2} / r^{2}\right)^{-(n-2) / 2} R\left(\frac{\partial}{\partial R}\left(r^{\mu_{j}} v_{j} / R\right)\right)^{2} d r d y<\infty,
$$

where $\partial / \partial R=R^{-1}(r \partial / \partial r+y \partial / \partial y)$. 
Notice also by definition we have

$$
v(r \omega, y)=\sum_{j=1}^{\infty} r^{\mu_{j}} v_{j}(r, y) \varphi_{j}(\omega)
$$

for $(r \omega, y) \in \mathbf{C} \cap B_{\rho}$.

Next we note (see the Appendix 1) that any solution $v_{j}$ of an equation of the form (9) which satisfies the integrability condition (10) is automatically real-analytic in the variables $r^{2}, y$; in fact

$v_{j}(r, y)=\sum_{k, \ell=0}^{\infty} a_{k, \ell}^{j} r^{2 k} y^{\ell}, r^{2}+y^{2}<(\theta \rho)^{2},\left|a_{k, \ell}^{j}\right| \leq(\theta \rho)^{-2 k-\ell}\left(\rho^{-2-\gamma} \int_{K_{\rho}} v_{j}^{2} d \mu\right)^{1 / 2}$

Also, the restriction (11) evidently implies that the total degree of $r^{\mu_{j}} a_{k, \ell}^{j} r^{2 k} y^{\ell}$ (for $a_{k, \ell}^{j} \neq 0$ ) cannot be strictly less than 1 ; that is, $\mu_{j}+2 k+\ell \geq 1$ for any $k, \ell$ such that $a_{k, \ell}^{j} \neq 0$.

Thus by virtue of (12), the solution $v$ has an expansion of the form

$$
v=\sum_{j} r^{\mu_{j}} \sum_{k, \ell} a_{k, \ell}^{j} r^{2 k} y^{\ell} \varphi_{j}(\omega)
$$

where

$$
\mu_{j}+2 k+\ell \geq 1 \quad \forall j, k, \ell \text { such that } a_{k, \ell}^{j} \neq 0 .
$$

Our main concern will be the possibility that $\mu_{j}+2 k+\ell=1$ here. We proceed to analyse this possibility. In case $2 k+\ell=0$, we have $2 k+\ell+\mu_{j}=1$ if and only if $\mu_{j}=1$; then in view of our integrability assumption $\ddagger$ (b) the only non-zero terms in the expression (13) with $2 k+\ell=0$ and $\mu_{j}+2 k+\ell=1$ have the form $r \sum_{j=1}^{N} a_{j} \varphi_{j}(\omega)$, with $r \varphi_{j}(\omega)$ generated by rotations as in Example 1 above.

For $2 k+\ell=1$ we have $\mu_{j}+2 k+\ell=1$ if and only if $\mu_{j}=0, \ell=1, k=0$, and by the other part of assumption $\ddagger$ (b) the only non-zero terms in the expression (13) with $\ell=1, k=0$ have the form $y \sum_{j=1}^{Q} b_{j} \varphi_{j}$, with $\varphi_{j}(\omega)$ generated by translations as in Example 2 above.

For $2 k+\ell \geq 2$ we get $2 k+\ell+\mu_{j}=1$ only for $\mu_{j}=1-(2 k+\ell)$, but by direct computation this corresponds to the case

$$
\lambda_{j}=-q(n-3-q), 1 \leq q \leq \frac{n-3}{2}, q(=2 k+\ell-1) \text { an integer }
$$


and for $q<(n-3) / 2$, this is excluded by the eigenvalue restriction of condition $\ddagger(\mathrm{c})$. On the other hand if $q=(n-3) / 2$ then $\lambda_{j}=-(n-3)^{2} / 4$, giving $\lambda_{1} \leq-(n-3)^{2} / 4$ and thus contradicting the assumption that $\mathbf{C}_{0}$ is strictly stable. Thus the possibility $\mu_{j}+2 k+\ell=1,2 k+\ell \geq 2$ is entirely excluded.

Thus, in summary, the expansion for $v$ in (13) has the form

$$
v(r \omega, y)=r \sum_{j=1}^{N} a_{j} \varphi_{j}(\omega)+y \sum_{j=1}^{Q} b_{j} \psi_{j}(\omega)+R(r \omega, y),
$$

where each $r \varphi_{j}$ is generated by rotations as in Example 1, each $\psi_{j}$ generated by translations as in Example 2, $a_{j}, b_{j}$ are constants, $\varphi_{j}, \psi_{j}$ are orthonormal in $L^{2}(\Sigma)$, and $R$ satisfies

$$
\rho^{-n-2} \int_{B_{\rho}^{n+1}}|R(r \omega, y)|^{2} \leq C \rho^{1+\alpha} \quad \forall \rho \in(0,1 / 2),
$$

where $C, \alpha>0$ depend only on $\Sigma$; also, because $\|v\|_{L^{2}\left(B_{\rho}\right)} \leq 1$ by construction, we must have the bounds

$$
\left|a_{j}\right|,\left|b_{j}\right| \leq C
$$

with $C$ depending only on $\Sigma$.

Notice that (14), (15), (16) can be written in the form

$$
v(r \omega, y)=(\nu(\omega), 0) \cdot A \cdot(r \omega, y)+R(r \omega, y),
$$

where $A$ is a (constant) skew-symmetric linear transformation of $\mathbf{R}^{n+1}$ and

$$
\left\{\begin{aligned}
\rho^{-n-2} \int_{B_{\rho}^{n+1}}|R(r \omega, y)|^{2} & \leq C \rho^{1+\alpha}, \forall \rho \in(0,1 / 2) \\
|A| & \leq C
\end{aligned}\right.
$$

with $C$ depending only on $\Sigma$. (Notice that here we use the fact that the unit normal of $\mathbf{C}=\mathbf{C}_{0} \times \mathbf{R}$ is given by $\bar{\nu}=\left(\nu^{1}, \ldots, \nu_{n+1}\right)$, where $\nu^{n+1} \equiv 0$, so that $y \nu_{j}(\omega) \equiv y \nu_{j}-\omega^{j} \nu_{n+1}$ for $j=1, \ldots, n$.)

Now the term $(\nu(\omega), 0) \cdot A \cdot(r \omega, y)$ appearing in (14) is a solution of the equation $L_{\mathbf{C}} v=0$ which is generated by rotations of the $(n+1)$-dimensional cone $\mathbf{C}$ in the same way that the $r \varphi_{j}$ are generated by rotations of the $n$ dimensional cone $\mathbf{C}_{0}$ as described in Example 1. (Here the 1-parameter family of rotations is $q_{t}=\exp t A$.) 
We therefore have that there is a sequence of orthogonal transformations $q_{k}\left(=\exp \sigma_{k} A\right)$ for a suitable sequence $\left.\sigma_{k} \downarrow 0\right)$ of $\mathbf{R}^{n+1}$ such that if $\widetilde{U}_{k}, \widetilde{u}_{k}, \widetilde{v}_{k}$ denote the quantities corresponding to $U_{k}, u_{k}, v_{k}, d_{k}$ in case $M$ is replaced by $q_{k}^{-1}$, then for suitable fixed $\theta \in\left(0, \frac{1}{2}\right)$ (depending only on $\Sigma$ )

$$
\theta^{-n-2} \int_{B_{\theta} \cap U_{k} \cap\{(x, y):|x| \geq \sigma\}} \tilde{u}_{k}^{2} \leq \frac{1}{4} \int_{M_{k} \cap B_{1}} d_{k}^{2}
$$

for any fixed $\sigma \in(0,1)$ and all sufficiently large $k$ (depending on $\sigma$ ), by virtue of the fact that the $C^{1}$ norm of $u_{k}$ over the set $U_{k}$ converges to zero, and, by a discussion like that in Example 1 above (but with $\mathbf{C}$ in place of $\mathbf{C}_{0}$ ), we have that the ratio $\delta_{k}^{-1} u_{k}$ converges (in $C^{1}$ away from the axis $|x|=0$ ) to $v$, with $v$ given by (17), whereas $\delta_{k}^{-1} \widetilde{u}_{k}$ converges locally away from the axis $\{(x, y):|x|=0\}$ to a function $\widetilde{v}$, where $\widetilde{v}$ has the same form as $v$ in (17) but with $A=0$; therefore we conclude (19) as required for suitable $\theta \in\left(0, \frac{1}{2}\right)$ depending only on $\Sigma$.

Then, in view of the Corollary 2.4, we have actually

$$
\theta^{-n-2} \int_{M_{k} \cap B_{\theta}} \widetilde{d}_{k}^{2} \leq \frac{1}{2} \int_{M_{k} \cap B_{1}} d_{k}^{2}
$$

and in view of the arbitrariness of the sequences $M_{k}, \epsilon_{k}$ used in the above argument, we see in particular (by applying the above discussion to homotheties of $\mathrm{M})$ that the following result is established: If $\rho \in(0.1]$ and if $\rho^{-1} M \in \mathcal{N}_{\epsilon}(\mathbf{C})$, then

$$
(\theta \rho)^{-n-2} \int_{B_{\theta \rho} \cap M} d_{q}^{2} \leq \frac{1}{2} \rho^{-n-2} \int_{B_{\rho} \cap M} d^{2},
$$

where $d_{q}(x, y)=\operatorname{dist}\{(x, y), q(\mathbf{C})\}$ for $(x, y) \in M$.

Notice that this can of course be applied with any rotation of $\mathbf{C}$ in place of $\mathbf{C}$, and hence the above can be iterated arbitrarily many times, and under the hypotheses of the lemma (with $\epsilon / 2$ in place of the $\epsilon$ which we found above) we actually conclude that there is a sequence of orthogonal transformations $q_{k}$ of $\mathbf{R}^{n+1}$ such that

$$
\left(\theta^{k} \rho\right)^{-n-2} \int_{M \cap B_{o^{k} \rho}} d_{k}^{2} \leq 2^{-k} \rho^{-n-2} \int_{B_{\rho} \cap M} d^{2},
$$


where $d_{k}(x, y) \equiv \operatorname{dist}\left\{(x, y), q_{k}(\mathbf{C})\right\}$. Notice that (since $\left|M \cap B_{\sigma}\right| \geq C \sigma^{n}$ for any $\sigma<\rho$ ) this directly implies (by the triangle inequality) that $\left|q_{k}-q_{k+1}\right|^{2} \leq$ $C 2^{-k} \rho^{-n-2} \int_{M \cap B_{\rho}} d^{2}$, and hence that there exists $q_{*}=\lim q_{k}$ with

$$
\left|q_{*}-q_{k}\right|^{2} \leq C 2^{-k} \rho^{-n-2} \int_{M \cap B_{\rho}} d^{2}
$$

and

$$
\left(\theta^{k} \rho\right)^{-n-2} \int_{M \cap B_{0^{k} \rho}} d_{*}^{2} \leq C 2^{-k} \rho^{-n-2} \int_{B_{\rho} \cap M} d^{2}, \forall k \geq 1
$$

where $d_{*} \equiv d_{q_{*}}$. Then since any $\sigma \in(0, \rho)$ lies in one of the intervals $\left[\theta^{k+1} \rho, \theta^{k} \rho\right)$, we finally conclude that

$$
\sigma^{-n-2} \int_{M \cap B_{\sigma}} d_{*}^{2} \leq C(\sigma / \rho)^{\alpha} \rho^{-n-2} \int_{M \cap B_{\rho}} d^{2}
$$

where $C>0$ and $\alpha \in(0,1)$ are fixed constants depending only on $\Sigma$.

From the above argument it is easy to see that we actually have the following stronger version of Theorem 1:

Theorem 1'. If the hypotheses are as in Theorem 1, there is an orthogonal transformation $q$ of $\mathbf{R}^{n+1}$ such that $\|q-\mathrm{id}\| \leq C \epsilon$ and

(*) $\quad \sigma^{-n-2} \int_{M \cap B_{\boldsymbol{\sigma}}} \widetilde{d}^{2} \leq C(\sigma / \rho)^{\alpha} \rho^{-n-2} \int_{M \cap B_{\rho}} d^{2}, \quad \tilde{d}(X)=\operatorname{dist}(X, q(\mathbf{C}))$,

where $C>0$ and $\alpha \in(0,1)$ are fixed constants depending only on $\Sigma$. In particular the convergence is $C^{2}$ away from the singular axis $q(\{0\} \times \mathbf{R})$; the convergence is also locally in the Hausdorff distance sense on all of $\mathbf{R}^{n+1}$.

Proof. First, the inequality $(*)$ is exactly the inequality (21) established in the proof of Theorem 1. By virtue of the argument in the proof of Lemma 1.4 in $\S 1$, we deduce from * that there is a domain $W \subset \mathbf{C}$ with

$$
W \cap B_{\sigma} \supset \mathbf{C} \cap B_{\sigma} \backslash\left\{(x, y) \in \mathbf{C}:|x| \leq C \sigma^{1+\alpha} \epsilon^{1 / 2}\right\}
$$

for each $\sigma \in(0,1)$, and a $u \in C^{\infty}(W)$ such that

$$
|x|^{-1}|u(x, y)|+|\nabla u(x, y)| \leq C\left(|x|^{2}+y^{2}\right)^{\alpha / 2} \epsilon^{1 / 2} \text { for }(x, y) \in W
$$


and

$$
M \cap B_{1 / 2} \backslash\{(x, y)+u(x, y) \nu(\omega):(x, y) \in W\} \subset\left\{(x, y):|x| \leq C|y|^{1+\alpha} \epsilon^{1 / 2}\right\} .
$$

It now directly follows from (2) and (3), together with the standard local interior $C^{k}$ estimates [GT] for the solutions of quasilinear equations with small $C^{1}$-norm, that $M$ converges in the required $C^{2}$-sense and also in the weak sense of currents. The fact that the convergence is also in the Hausdorff distance sense follows from general facts about convergence of stationary submanifolds (or more generally varifolds) to their tangent cones-see e.g. [AW], [SL3].

\section{Concluding Remarks}

We first note here that essentially only notational changes are required in order to extend Theorems 1, 2 to handle the case when $\mathbf{C}=\mathbf{C}_{0} \times \mathbf{R}^{m}$ with $m \geq 2$ (rather than $\mathbf{C}_{0} \times \mathbf{R}$ ). For example (letting $\mathcal{M}^{m}$ and $\mathcal{N}_{\epsilon}^{m}$ correspond in the obvious way to $\mathcal{M}$ and $\mathcal{N}_{\epsilon}$ in case we have $\mathbf{C}=\mathbf{C}_{0} \times \mathbf{R}^{m}$ in place $\mathbf{C}_{0} \times \mathbf{R}$ ), the analogue of Theorem 1 in this more general setting is:

Theorem 4.1. Suppose $\mathbf{C}_{0}$ satisfies the conditions $\ddagger$. There is $\epsilon=\epsilon\left(\mathbf{C}_{0}, m\right)>$ 0 such that if $M \in \mathcal{N}_{\epsilon}^{m}(\mathbf{C})$ with $\epsilon \leq \epsilon_{0}$, then there is a rotation $\widetilde{\mathbf{C}}=q_{\sharp} \mathbf{C}$ of $\mathbf{C}$ such that $\widetilde{\mathbf{C}}$ is the unique tangent cone of $M$ at zero, so that $(\rho)^{-1} M \rightarrow \mathbf{C}$ as $\rho \downarrow 0$. The convergence is also in the $C^{2}$ sense away from $q_{\sharp}\left(\{0\} \times \mathbf{R}^{m}\right)$ and locally in the Hausdorff distance sense.

Finally we mention that these results have close analogues in the theory of energy minimizing maps.

\section{Appendix 1}

Here we use the notation

$$
\begin{aligned}
K_{\rho} & =\left\{(r, y): r>0, r^{2}+y^{2}<\rho^{2}\right\} \\
K_{\rho}\left(r_{0}, y_{0}\right) & =\left\{(r, y): r>0,\left(r-r_{0}\right)^{2}+\left(y-y_{0}\right)^{2}<\rho^{2}\right\} \\
\nabla w & =\left(\frac{\partial w}{\partial r}, \frac{\partial w}{\partial y}\right) \\
d \mu & =r^{\gamma} d r d y
\end{aligned}
$$


where $\gamma \in[1, \infty)$ is a given real constant.

We want to prove that if $w$ is a $W_{\text {loc }}^{1,2}\left(K_{\rho}\right)$ solution of

$$
r^{-\gamma} \frac{\partial}{\partial r}\left(r^{\gamma} \frac{\partial w}{\partial r}\right)+\frac{\partial^{2} w}{\partial y^{2}}=0
$$

which satisfies the integrability condition

$$
\int_{K_{\rho}}\left(r^{-2} w^{2}+|\nabla w|^{2}\right) d \mu<\infty
$$

then $w$ automatically extends to a real-analytic function of the variables $r^{2}, y$ for $r^{2}+y^{2}<\rho$ and in fact has the expansion

$w(r, y)=\sum_{j, \ell=0}^{\infty} a_{j, \ell} r^{2 j} y^{\ell}, r^{2}+y^{2}<(\theta \rho)^{2}, \quad\left|a_{j, \ell}\right| \leq(\theta \rho)^{-2 j-\ell}\left(\rho^{-\gamma-2} \int_{K_{\rho}} w^{2} d \mu\right)^{1 / 2}$,

where $\theta \in(0,1)$ is a fixed constant depending only on $\gamma$. First note that when $\gamma=1$ the equation (1) is just the assertion that $w$ is harmonic function of the variables $\left(x_{1}, x_{2}, y\right) \in \mathbf{R}^{2} \backslash\{0\} \times \mathbf{R}$, such that, if $w$ is expressed as a function of the cylindrical coordinates $(r \cos \theta, r \sin \theta, y)$, then $w$ is independent of $\theta$. Further, the integrability condition guarantees that $w$ is in $W^{1,2}$ on the whole ball $B_{\rho}^{3}$ and hence we deduce that in fact $w$ is weakly harmonic in the ball $B_{\rho}^{3}$. It is then standard that $w$ is real-analytic, and, since $w$ is independent of $\theta, w$ has a convergent power series in the variables $r^{2}, y$ for $r^{2}+y^{2}<\rho$. (Notice that the same argument applies generally to the case when $\gamma$ is a positive integer.)

Thus we may assume in the remaining discussion that $\gamma>1$. To prove the required analyticity in this case, we first note that the equation (1) has the weak form

$$
\int_{K_{\rho}} \nabla w \cdot \nabla \zeta d \mu=0
$$

valid at least if $\zeta \in W^{1,2}\left(K_{\rho}\right)$ with support $\zeta \subset K_{\rho}$. We claim that in fact, because of the integrability condition $(2),(4)$ actually holds for any $\zeta \in W_{\text {loc }}^{1,2}\left(K_{\rho}\right)$ satisfying

$$
\int_{K_{\rho}}\left(r^{-2} \zeta^{2}+|\nabla \zeta|^{2}\right)<\infty, \text { support } \zeta \cap\left\{(r, y): r^{2}+y^{2}=\rho^{2}\right\}=\emptyset .
$$

(Notice in particular that this means that there is no requirement that $\zeta(r, y)$ vanish in any sense as $r \downarrow 0$.) The fact that (4) holds for all $\zeta$ as in (5) 
is easily checked by an approximation argument (using cut-off functions to approximate $\zeta$ as in (5) by a sequence $\zeta_{k}$ with $\zeta_{k} \equiv 0$ in a neighbourhood of the axis $r=0$ ).

Before proceeding further we note that for any $\zeta$ as in (5) we have the inequality

$$
\int_{K_{\rho}} r^{-2} \zeta^{2} d \mu \leq C \int_{K_{\rho}}|\nabla \zeta|^{2} d \mu
$$

by virtue of a simple integration by parts argument based on the fact that $\gamma>1$.

Now for $\theta \in(0,1), \sigma \in(0, \rho)$ and $\left(r_{0}, y_{0}\right) \in K_{\rho-\sigma}$ we may choose $\zeta=\varphi^{2} w$ in (4), where $\varphi \equiv 1$ on the ball $K_{\theta \sigma}\left(r_{0}, y_{0}\right), \varphi \equiv 0$ in $K_{\rho} \backslash K_{\sigma}\left(r_{0}, y_{0}\right)$, and $|\nabla \varphi| \leq 2(1-\theta)^{-1} \sigma^{-1}$. This gives (after a standard application of Cauchy's inequality)

$$
\int_{K_{\theta \sigma}\left(r_{0}, y_{0}\right)}|\nabla w|^{2} d \mu \leq C(1-\theta)^{-2} \sigma^{-2} \int_{K_{\sigma}\left(r_{0}, y_{0}\right)} w^{2} d \mu
$$

where $C$ is a fixed constant not depending on $\rho, \sigma, \theta$.

Now if $\sigma \in(0, \rho)$ and $\left(r_{0}, y_{0}\right) \in K_{\rho-\sigma}$, and if $r_{0}>\sigma$, then the standard uniformly elliptic theory tells us that

$$
\sup _{K_{\sigma / 2}\left(r_{0}, y_{0}\right)} w^{2} \leq C \sigma^{-\gamma-2} \int_{K_{\sigma}\left(r_{0}, y_{0}\right)} w^{2} d \mu .
$$

But since $w^{2}$ is a subsolution of the equation (1) we know from [SL2] that the function $\varphi(\sigma) \equiv \sigma^{-2-\gamma} \int_{K_{\sigma}\left(0, y_{0}\right)} w^{2} d \mu$ is an increasing function of $\sigma$, so we conclude for suitable constant $C$ depending only on $\gamma$ that

(8) $\sup _{K_{\sigma}\left(r_{0}, y_{0}\right)} w^{2} \leq C \tau^{-\gamma-2} \int_{K_{\tau}\left(r_{0}, y_{0}\right)} w^{2} d \mu, 0<2 \sigma<\tau<\rho, r_{0}^{2}+y_{0}^{2}<(\rho-\tau)^{2}$.

Next, for $0<|h|<\theta \sigma$ let $w_{h}(r, y)=h^{-1}(w(r, y+h)-w(r, y))$, and note that $w_{h}$ is a solution of (4) provided support $\zeta \subset K_{\rho-|h|}$, and hence (7) holds with $w_{h}$ in place of $w$, provided $\sigma<\rho-|h|$. Letting $h \rightarrow 0$, we thus deduce that $w^{(1)} \equiv \partial w / \partial y$ is in $W_{\mathrm{loc}}^{1,2}\left(K_{\rho}\right)$, and satisfies

$$
\int_{K_{\theta_{\sigma}}\left(r_{0}, y_{0}\right)}\left(r^{-2}\left(w^{(1)}\right)^{2}+\left|\nabla w^{(1)}\right|^{2}\right) d \mu \leq C(1-\theta)^{-2} \sigma^{-2} \int_{K_{\sigma}\left(r_{0}, y_{0}\right)}\left(w^{(1)}\right)^{2} d \mu ;
$$


here we have used (6) in order to justify the inclusion of the term $r^{-2}\left(w^{(1)}\right)^{2}$ on the left. Since $\left|\nabla w^{(1)}\right|^{2} \geq\left(w^{(2)}\right)^{2}$, this implies in particular that

$$
\int_{K_{\sigma_{\sigma}}\left(r_{0}, y_{0}\right)}\left(w^{(2)}\right)^{2} \leq C(1-\theta)^{-2} \sigma^{-2} \int_{K_{\sigma}\left(r_{0}, y_{0}\right)}\left(w^{(1)}\right)^{2} d \mu .
$$

Proceeding inductively we have that each $w^{(k)}$ is a solution of the equation with

$$
\int_{K_{\theta \sigma}\left(r_{0}, y_{0}\right)}\left(r^{-2}\left(w^{(k)}\right)^{2}+\left|\nabla w^{(k)}\right|^{2}\right) d \mu \leq C(1-\theta)^{-2} \sigma^{-2} \int_{K_{\sigma}\left(r_{0}, y_{0}\right)}\left(w^{(k)}\right)^{2} d \mu
$$

and

$$
\int_{K_{\theta \sigma}\left(r_{0}, y_{0}\right)}\left(w^{(k+1)}\right)^{2} d \mu \leq C(1-\theta)^{-2} \sigma^{-2} \int_{K_{\sigma}\left(r_{0}, y_{0}\right)}\left(w^{(k)}\right)^{2} d \mu
$$

for each $k=0,1, \ldots$ Selecting $\theta=k /(k+1)$ and iterating this last inequality, we get

$$
\int_{K_{\sigma /(k+1)}\left(r_{0}, y_{0}\right)}\left(w^{(k)}\right)^{2} d \mu \leq C^{k}(k !)^{2} \sigma^{-2 k} \int_{K_{\rho-\sigma}} w^{2} d \mu
$$

for suitable $C$ independent of $k$. By combining this with (8) (with $w^{(k)}$ in place of $w$ ) and using $\sigma=\rho / 4$ we then conclude that

$$
\left|w^{(k)}(r, y)\right| \leq C^{k} k ! \rho^{-k}\left(\rho^{-\gamma-2} \int_{K_{\rho}} w^{2} d \mu\right)^{1 / 2}
$$

for any $(r, y) \in K_{\rho / 4}$.

Now let $L_{r}=r^{-\gamma}\left(\partial\left(r^{\gamma} \partial / \partial r\right) / \partial r\right)$, and notice that the equation (1) implies

$$
L_{r} w=-w^{(2)} \text { on } K_{\rho}
$$

so that (since $\partial / \partial y$ commutes with $L_{r}$ )

$$
L_{r}^{j} w^{(\ell)}=(-1)^{j} w^{(2 j+\ell)} \text { for each pair of integers } j, \ell \geq 0
$$

and hence (9), (10) imply

$$
\left\{\begin{array}{l}
\sup _{K_{\rho / 4}}\left|L_{r}^{j}\left(\frac{\partial}{\partial y}\right)^{\ell} w\right| \leq C^{2 j+\ell}(2 j) !(\ell) ! \rho^{-2 j-\ell}\left(\rho^{-\gamma-2} \int_{K_{\rho}} w^{2} d \mu\right)^{1 / 2} . \\
r^{-1} L_{r}^{j}\left(\frac{\partial}{\partial y}\right)^{\ell} w, \nabla\left(L_{r}^{j}\left(\frac{\partial}{\partial y}\right)^{\ell} w\right) \in L^{2}\left(\mu, K_{\sigma}\right)
\end{array}\right.
$$

for $\sigma<\rho / 8$. The required expansion (3) of $w$ follows from this. Indeed, if $\psi$ is a smooth function in $K_{\rho}$ with $r^{-1} \psi$ if $|\nabla \psi|$ both in $L^{2}(\mu)$ on $K_{\rho}$ and if 
$\sup _{K_{\rho}}\left|L_{r} \psi\right| \leq \beta$ ( $\beta$ a given constant), then direct integration shows that then for each $\sigma<r / 2<\rho / 4$

$$
r^{\gamma}\left|\frac{\partial \psi}{\partial r}\right|(r, y) \leq C \sigma^{-1} \int_{\sigma / 2}^{\sigma} \tau^{\gamma}\left|\frac{\partial \psi}{\partial r}\right|(\tau, y) d \tau+C \beta r^{\gamma+1}
$$

and since the first term on the right $\rightarrow 0$ in $L^{1}(-\rho / 4,-\rho / 4)$ (in the $y$-variable) we can choose $\sigma=\sigma_{j} \downarrow 0$ to ensure that it converges pointwise a.e. to zero; thus we conclude

$$
\left|\frac{\partial \psi}{\partial r}\right|(r, y) \leq C \beta r
$$

with $C$ fixed depending only on $\gamma$. By another direct integration we then also deduce that $\psi$ extends continuously to $\bar{K}_{\rho / 4}$ and

$$
\left|\psi(r, y)-\psi\left(0_{+}, y\right)\right| \leq C \beta r^{2} .
$$

Notice that using these general facts on the functions $\psi=L_{r}^{j} w^{(\ell)}$ (which we can do by virtue of (11)), we can easily prove inductively that then

$$
w \in C^{\infty}\left(\bar{K}_{\rho / 8}\right) \text { and }\left.\frac{\partial L^{j} w(r, y)}{\partial r}\right|_{r=0} \equiv 0, \quad|y| \leq \rho / 8
$$

We claim that in addition we have

$$
\sup _{K_{\theta_{\rho}}}\left|\frac{\partial^{j}}{\partial r^{j}} \frac{\partial^{\ell}}{\partial y^{\ell}} w\right| \leq C^{\ell+j} \ell ! j !\left(\rho^{-\gamma-2} \int_{K_{\rho}} w^{2} d \mu\right)^{1 / 2}
$$

for some fixed $C>0$ and $\theta \in(0,1 / 8)$ depending only on $\gamma$. Indeed one first checks the validity of this with $j=0$ and $\ell$ arbitrary directly from (11); then use a further inductive argument based on repeated integration (with respect to the $r$-variable) of (11) (this time with $\ell=0$ ), making use of $(12)$, to deduce that for each $j \geq 0$ and each $y \in(-\rho / 8, \rho / 8)$

$$
\sup _{K_{\theta \rho}} r^{-2 j-2}\left|w(r, y)-\sum_{k=0}^{j} \beta_{k}^{(j)}\left(L_{r}^{k} w\right)\left(0_{+}, y\right) r^{2 k}\right| \leq 2^{-j}\left(\rho^{-\gamma-2} \int_{K_{\rho}} w^{2} d \mu\right)^{1 / 2}
$$

for some $\theta=\theta(\gamma) \in(0,1)$, where $\beta_{k}^{(j)}$ is a constant depending only on $k, \gamma, j$ such that $\left|\beta_{k}^{(j)}\right| \leq C^{k} /(2 k) ! \forall k, j \geq 0$. Now, by virtue of $(11),\left(L_{r}^{k} w\right)\left(0_{+}, y\right)$ are real-analytic functions of $y$ and satisfy

$$
\sup _{|y|<\rho / 8}\left|\frac{\partial^{\ell}}{\partial y^{\ell}} L_{r}^{k}\left(0_{+}, y\right)\right| \leq C^{\ell+k}(2 k) ! \ell !\left(\rho^{-\gamma-2} \int_{K_{\rho}} w^{2} d \mu\right)^{1 / 2}
$$


(13) (and hence (3)) now easily follows from (14) and (15).

\section{REFERENCES}

[AA] Allard, W. and Almgren, F., On the radial behavior of minimal surfaces and the uniqueness of their tangent cones, Ann. of Math. 113 (1981), 215-265.

[AW] Allard, W., On the first variation of a varifold, Ann. of Math. 95 (1972), 417-491

[CHS] Caffarelli, L., Hardt, R. and Simon, L., Minimal surfaces with isolated singularities, Manuscripta Math. 48 (1984), 1-18.

[FH] Federer, H., Geometric Measure Theory, Springer-Verlag, Berlin-HeidelbergNew York 1969.

[FK] Ferus, D. and Karcher, H., Non-rotational minimal spheres and minimizing cones, Comment. Math. Helv. 60 (1985), 247-269.

[GT] Gilbarg, D. and Trudinger, N., Elliptic Partial Differential Equations of Second Order, ( $2^{\text {nd }}$ Edition), Springer-Verlag, Berlin-Heidelberg-New York 1983.

[HS] Hardt, R. and Simon, L., Area minimizing hypersurfaces with isolated singularities, J. Reine. Angew. Math. 362 (1985), 102-129.

[L] Lawson, H. B., The equivariant Plateau problem and interior regularity, Trans. Amer. Math. Soc. 173 (1973), 231-149.

[SS] Simon, L. and Solomon, B., Minimal hypersurfaces asymptotic to quadratic cones, Invent. Math. 86 (1986), 535-551

[SL1] Simon, L., Asymptotics for a class of non-linear evolution equations, with applications to geometric problems, Ann. of Math. 118 (1983), 525-572.

[SL2] Simon, L., Entire solutions of the minimal surface equation, J. Diff. Geom. 30 (1989), 643-688.

[SL3] Simon, L., Lectures on Geometric Measure Theory, Proceedings of the Centre for Mathematical Analysis, Australian National University, 3 (1983).

[SL4] Simon, L., On the singularities of harmonic maps, In preparation.

[SL5] Simon, L., Cylindrical tangent cones and the singular set of minimal submanifolds, (preprint 1991-To appear in J. Diff. Geom.)

[SJ] Simons, J., Minimal varieties in Riemannian manifolds, Ann. of Math. 88 (1968), 62-105.

[SB1] Solomon, B., The harmonic analysis of cubic isoparimetric minimal hypersurfaces I, to appear in Amer. J. Math..

[SB2] Solomon, B., The harmonic analysis of cubic isoparimetric minimal hypersurfaces II, to appear in Amer. J. Math..

Stanford University, U. S. A.

ReCEIVED MaRCh 2, 1993. 